\title{
VARIATIONAL INTEGRALS WITH A WIDE RANGE OF ANISOTROPY
}

\author{
M. BILDHAUER, M. FUCHS, AND X. ZHONG
}

\begin{abstract}
Anisotropic variational integrals of $(p, q)$-growth are considered. For the scalar case, the interior $C^{1, \alpha}$-regularity of bounded local minimizers is proved under the assumption that $q \leq 2 p$, and a famous counterexample of Giaquinta is discussed. In the vector case, some higher integrability result for the gradient is obtained.
\end{abstract}

\section{$\S 1$. INTRODUCTION}

Roughly speaking, an anisotropic variational integral of the type $J[u]=\int_{\Omega} f(\nabla u) \mathrm{d} x$, defined for functions $u$ : $\Omega \rightarrow \mathbb{R}^{N}$ on some bounded domain $\Omega \subset \mathbb{R}^{n}, n \geq 2$, is characterized via a growth condition such as

$$
a|Q|^{p}-b \leq f(Q) \leq A|Q|^{q}+B,
$$

where $a, b, A, B$ denote positive constants, $1<p<q<\infty$ are given exponents, and $Q$ is an arbitrary matrix in $\mathbb{R}^{n N}$. A natural space for the local minimizers is the class of functions $u$ in $W_{p}^{1}\left(\Omega ; \mathbb{R}^{N}\right)$ such that $\int_{\Omega^{\prime}} f(\nabla u) \mathrm{d} x<\infty$ for each subregion $\Omega^{\prime} \Subset \Omega$; we are interested in the regularity properties of local minimizers $u$, which means that we ask about the higher integrability of $\nabla u$, the Hölder continuity of $u$, or even the Hölder continuity of $\nabla u$ provided that $f$ satisfies some additional smoothness and convexity assumptions. In general, the hope for positive results increases in the scalar case, but the counterexamples of [Gi2] and (later) of [Ho] show that even for $N=1$, unbounded minimizers exist if $q$ is too big with respect to $p$.

On the contrary, there is a long list of authors investigating various aspects of the regularity theory; among others, we mention the works of Acerbi and Fusco [AF], Fusco and Sbordone [FS], Marcellini [Ma1, Ma2], Choe [Ch], and the papers [ELM1, ELM2] of Esposito, Leonetti and Mingione, where the interested reader can also find further references.

Typically, in the above-mentioned publications, either a bound of the form

$$
q<c(n) p
$$

with $c(n)>1$, but $c(n) \rightarrow 1$ as $n \rightarrow \infty$, is required, or a dimensionless restriction such as

$$
q<p+2
$$

occurs together with the assumption that $u$ is locally bounded. Then, as a first step, it is shown that actually $\nabla u$ is in the space $L_{\text {loc }}^{q}\left(\Omega ; \mathbb{R}^{n N}\right)$. In its turn, this result is used at the second step to prove the $C^{1, \alpha}$-regularity in the scalar case or in the vector case under an additional structure condition, whereas in the general vector setting the partial $C^{1, \alpha}$-regularity is established. Of course, to do this,(1.1) must be replaced by a stronger

2000 Mathematics Subject Classification. Primary 35J20; Secondary 35A15, 49Q20.

Key words and phrases. Anisotropic problems, regularity of minimizers. 
condition: for example, one may assume that $f: \mathbb{R}^{n N} \rightarrow[0, \infty)$ is of class $C^{2}$ and satisfies

$$
\lambda\left(1+|Q|^{2}\right)^{\frac{p-2}{2}}|Z|^{2} \leq D^{2} f(Q)(Z, Z) \leq \Lambda\left(1+|Q|^{2}\right)^{\frac{q-2}{2}}|Z|^{2}, \quad Q, Z \in \mathbb{R}^{n N},
$$

where $\lambda$ and $\Lambda$ are positive constants. Clearly (1.4) implies (1.1); moreover, the first inequality in (1.4) shows that $f$ is strictly convex.

In this note we take a closer look at the counterexamples mentioned above. Giaquinta's example works with the choice $p=2, q=4, n \geq 6$ : he considered the variational integral

$$
J[u]=\int\left[\sum_{i=1}^{n-1}\left(\partial_{i} u\right)^{2}+\frac{1}{2}\left(\partial_{n} u\right)^{4}\right] \mathrm{d} x,
$$

for which, provided $n \geq 6$,

$$
u(x):=\sqrt{\frac{n-4}{24}} \frac{x_{n}^{2}}{\left(\sum_{i=1}^{n-1} x_{i}^{2}\right)^{\frac{1}{2}}}
$$

is of finite energy and satisfies the Euler-Lagrange equation, i.e., $u$ is a local $J$-minimizer. However, this singular minimizer is not bounded; in fact, in [FS] a sharp condition is proved under which we may expect unbounded minimizers. For $p=2$, this condition reads as

$$
n-1>\frac{2 q}{q-2}
$$

in particular, for $q=4$ we have singular unbounded solutions for $n \geq 6$. Note that the question about unbounded solutions is strongly related to the dimension $n$. On the other hand, as was mentioned above, the smoothness of the bounded solutions should follow from a dimensionless condition. For instance, up to now the existence of an anisotropic energy satisfying a condition such as (1.4) with $p=2, q=4$ and admitting locally bounded but nonsmooth local minimizers has not been clear. Observe that for $p=2$ and $q=4$ we have reached the limit case $q=p+2$ of condition (1.3); in accordance with [Bi, Theorem 5.4], the smoothness of locally bounded minimizers is only known under the stronger restriction $q<p+2$. In this paper, we are going to include the limit case of (1.3) in our considerations and relax (for $p \geq 2$ ) condition (1.3) to

$$
2 \leq p \leq q \leq 2 p,
$$

by employing the particular properties of the functionals under consideration. More precisely, we shall show that, actually, (1.5) together with some structural conditions imposed on $f$ is strong enough to ensure the usual smoothness properties of locally bounded solutions at least in the scalar case.

To discuss some details, we represent an element $Q$ of $\mathbb{R}^{n N}$ in the form $Q=\left(\tilde{Q}, Q_{n}\right)$, where

$$
\tilde{Q}:=\left(Q_{1}, \ldots, Q_{n-1}\right), \quad Q_{i} \in \mathbb{R}^{N}, i=1, \ldots, n .
$$

Then, a typical example we have in mind is given by

$$
f(Q)=\left(1+|\tilde{Q}|^{2}\right)^{\frac{p}{2}}+\left(1+\left|Q_{n}\right|^{2}\right)^{\frac{q}{2}} .
$$

In fact, we could also look at any decomposition

$$
Q=\left(Q^{(1)}, Q^{(2)}\right)
$$

of the matrix $Q$ into two submatrices $Q^{(i)}$ and consider $f$ with growth of order $p$ with respect to $Q^{(1)}$ and of order $q$ with respect to $Q^{(2)}$. Another model we could discuss is

$$
f(Q)=\sum_{i=1}^{n}\left(1+\left|Q_{i}\right|^{2}\right)^{\frac{p_{i}}{2}},
$$

where now $p:=\min p_{i}$ and $q:=\max p_{i}$. 
In order to keep our exposition simple, from now on we assume that $f \in C^{2}\left(\mathbb{R}^{n N}\right)$ can be written as (cf. (1.6))

$$
f(Q)=f_{1}(\tilde{Q})+f_{2}\left(Q_{n}\right)
$$

where

$$
\left\{\begin{array}{cl}
\lambda\left(1+|\tilde{Q}|^{2}\right)^{\frac{p-2}{2}}|\tilde{Z}|^{2} & \leq D^{2} f_{1}(\tilde{Q})(\tilde{Z}, \tilde{Z}) \quad \leq \Lambda\left(1+|\tilde{Q}|^{2}\right)^{\frac{p-2}{2}}|\tilde{Z}|^{2} \\
\lambda\left(1+\left|Q_{n}\right|^{2}\right)^{\frac{q-2}{2}}\left|Z_{n}\right|^{2} \leq D^{2} f_{2}\left(Q_{n}\right)\left(Z_{n}, Z_{n}\right) & \leq \Lambda\left(1+\left|Q_{n}\right|^{2}\right)^{\frac{q-2}{2}}\left|Z_{n}\right|^{2}
\end{array}\right.
$$

Note that (1.8) and (1.4) are different ellipticity conditions: obviously (1.4) does not imply (1.8), and if (1.8) is fulfilled, then we get a weaker form of (1.4) in which the exponent $p$ on the left-hand side must be replaced by 2 .

Moreover, we assume that

$$
\left\{\begin{aligned}
f_{1}(\tilde{Q}) & =f_{1}\left(Q_{1}, \ldots, Q_{n-1}\right)=g_{1}\left(\left|Q_{1}\right|, \ldots,\left|Q_{n-1}\right|\right), \\
f_{2}\left(Q_{n}\right) & =g_{2}\left(\left|Q_{n}\right|\right)
\end{aligned}\right.
$$

with $g_{2}$ monotone increasing and with $g_{1}$ monotone increasing with respect to each argument. The assumption (1.9) ensures the convex hull property (see, e.g., [BF3]); i.e., the global minimizer with respect to the Dirichlet boundary data $u_{0} \in L^{\infty}\left(\Omega ; \mathbb{R}^{N}\right)$ is also a bounded function. Therefore, it is reasonable to look at locally bounded local minimizers, where the fact that $u$ is a local minimizer means that

$$
J\left[u, \Omega^{\prime}\right]:=\int_{\Omega^{\prime}} f(\nabla u) \mathrm{d} x<\infty
$$

and $J\left[u, \Omega^{\prime}\right] \leq J\left[v, \Omega^{\prime}\right]$ for all $v$ such that $\operatorname{spt}(u-v) \subset \Omega^{\prime}$, and for all subdomains $\Omega^{\prime} \Subset \Omega$. Now we state our results.

Theorem 1.1. Suppose we are given exponents $2 \leq p \leq q<\infty$ satisfying (1.5). Let $f$ satisfy (1.7), (1.8), and (1.9), and let $u \in L_{\mathrm{loc}}^{\infty}\left(\Omega ; \mathbb{R}^{N}\right)$ denote a local minimizer. Then $\tilde{\nabla} u:=\left(\partial_{1} u, \ldots, \partial_{n-1} u\right) \in L_{\text {loc }}^{p+1}\left(\Omega ; \mathbb{R}^{(n-1) N}\right)$. Moreover, the function $\partial_{n} u$ is of class $L_{\mathrm{loc}}^{q+1}\left(\Omega ; \mathbb{R}^{N}\right)$.

Corollary 1.1. Under the assumptions of Theorem 1.1, assume additionally that $n-1<$ $p \leq q$. Then $u \in C^{0, \alpha}\left(\Omega ; \mathbb{R}^{N}\right)$ for some $0<\alpha<1$.

Remark 1.1. i) As was already noted, similar results can be obtained for decompositions different from that considered here.

ii) We may also consider functionals as above with an additional $x$-dependence (cf. $[\mathrm{BF} 4])$. Note that in the situation at hand we should not expect the Lavrentiev phenomenon.

iii) Modifying, for instance, the proof occurring in $[\mathrm{BFM}]$, we can also handle the case of degenerate ellipticity, which means that the 1 is dropped on the left-hand sides of the inequalities (1.8).

iv) In [Bi, Corollary 5.6], a partial regularity result was obtained under the ellipticity condition (1.4) and for the integrands $f(Q)=g\left(\left|Q_{1}\right|,\left|Q_{2}\right|, \ldots,\left|Q_{n}\right|\right)$, provided that $q<2+p$ and $q<p n /(n-2)$. It would be of interest to know whether partial regularity occurs in the above examples under a dimension-free condition on the exponents. This will be investigated in a separate paper.

v) If $p<2$ and if (1.4) is fulfilled, then better integrability results under the weaker assumption $q<p+2$ are valid even for functionals of a more general form than that required in (1.7); we refer to [Bi, Theorem 5.4]. Also, Theorem 1.2 and Corollary 1.2 below remain valid if $p<2, q<p+2$, and some modified conditions are imposed on $f$. 
Next, we turn our attention to the scalar case, for which we can improve the integrability of $\nabla u$. More precisely, the following statement is true.

Theorem 1.2. Let $N=1$, and let $f$ satisfy (1.7) and (1.8) together with $2 \leq p \leq q \leq 2 p$. If $u \in L_{\mathrm{loc}}^{\infty}(\Omega)$ is a local minimizer, then $\nabla u$ is in the space $L_{\mathrm{loc}}^{t}\left(\Omega ; \mathbb{R}^{n}\right)$ for any finite $t$.

Remark 1.2. i) Note that the structural condition (1.9) is not required in the scalar case.

ii) It would be desirable to extend Theorem 1.2 (including Corollary 1.2) to the vector situation studied in Theorem 1.1. This might be possible if, in addition to (1.9), $f_{1}$ also depends only on the modulus of the matrix, i.e., $f_{1}(\tilde{Q})=g_{1}(|\tilde{Q}|)$.

Corollary 1.2. In the scalar case, the locally bounded local minimizers of the variational integral $J[u, \Omega]=\int_{\Omega} f(\nabla u) \mathrm{d} x$ are of class $C^{1, \alpha}(\Omega)$ for any $0<\alpha<1$, provided that $f$ satisfies (1.7) and (1.8), and $2 \leq p \leq q \leq 2 p$.

Remark 1.3. i) We conjecture that the bound $q \leq 2 p$ is optimal, so that it would be of interest to find bounded solutions that are not of class $C^{1, \alpha}$ for a functional $J$ satisfying the hypotheses of Corollary 1.2 but with $q>2 p$, where $q$ can be chosen arbitrarily close to $2 p$.

ii) We would like to remark explicitly that, in general, sufficient conditions for regularity of the form (1.2) give better results for low dimensions $n$; for example, we mention the paper [FS], where for $n=2,3$ the bounds on $p$ and $q$ are less restrictive.

iii) As was mentioned above, in this paper we do not touch on the question of (partial) regularity in the vector case. We only note that for two-dimensional vector problems (i.e., $n=2$ and $N>1$ ) the condition $q<2 p$ is sufficient for interior $C^{1, \alpha}$-regularity even for the a priori unbounded local minimizers of the energy $\int_{\Omega} f(\nabla u) \mathrm{d} x$ with $f$ satisfying (1.4). We refer the reader to [BF2]. The method presented there should also cover the case where (1.4) is replaced by (1.8).

iv) If, for instance, $f(Q)=|\tilde{Q}|^{p}+\left|Q_{n}\right|^{q}$ with $p, q$ as above, then the solution is of class $W_{\infty, \text { loc }}^{1}$ (compare, e.g., [Bi, Theorem 5.22]).

Remark 1.4. For the scalar case, regularity results in the spirit of Corollary 1.2 were proved earlier in the paper [UU] by Ural'tseva and Urdaletova. As a byproduct of their considerations they showed that the locally bounded minimizers $u: \Omega \rightarrow \mathbb{R}$ of the variational integral $\int_{\Omega} \sum_{i=1}^{n}\left|\partial_{i} u\right|^{m_{i}} \mathrm{~d} x$ are actually Lipschitz on the compact subdomains of $\Omega$ provided that the exponents $m_{i}$ satisfy $m_{i}>1$ together with $2 m_{i}>\max \left\{m_{1}, \ldots, m_{n}\right\}$.

Our paper is organized as follows. In $\S 2$ we introduce an appropriate local regularization; i.e., we replace the integrand $f$ and the minimizer $u$ by suitable sequences $f_{k}$ and $u_{k}$, and prove a Caccioppoli-type inequality for the approximation. In the vector case this procedure is rather delicate since it is not quite clear if the test functions we use are really admissible. Then, the Caccioppoli-type inequality is employed to show that $\partial_{1} u, \ldots, \partial_{n-1} u$ are in the space $L_{\text {loc }}^{p+1}\left(\Omega ; \mathbb{R}^{N}\right)$. In $\S 3$ we study the scalar case and prove the first part of Theorem 1.2 by iteration. In $\S 4$ we deduce the fact that $u \in W_{t, \text { loc }}^{1}(\Omega)$ for any finite $t$, and we use this to get $u \in C^{1, \alpha}(\Omega)$.

\section{§2. Approximation And proof of Theorem 1.1}

Suppose that the assumptions of Theorem 1.1 are satisfied. Following familiar arguments, we introduce an appropriate local regularization: given $\epsilon>0$, we let $(u)_{\epsilon}$ denote 
the mollification of the local minimizer $u$ with radius $\epsilon$. We fix $x_{0} \in \Omega$ and a ball $B_{R}\left(x_{0}\right) \Subset \Omega$. Moreover, we define

$$
\delta:=\delta(\epsilon)=\frac{1}{1+\epsilon^{-1}+\left\|\nabla(u)_{\varepsilon}\right\|_{L^{q}\left(B_{R}\left(x_{0}\right) ; \mathbb{R}^{n N}\right)}^{2 q}}
$$

and put $f_{\delta}(Q)=\delta\left(1+|Q|^{2}\right)^{q / 2}+f(Q)$. Finally, let $u_{\delta} \in(u)_{\varepsilon}+\stackrel{\circ}{W_{q}^{1}}\left(B_{R}\left(x_{0}\right) ; \mathbb{R}^{N}\right)$ denote a unique solution of the problem

$$
J_{\delta}\left[w, B_{R}\left(x_{0}\right)\right]:=\int_{B_{R}\left(x_{0}\right)} f_{\delta}(\nabla w) \mathrm{d} x \rightarrow \min \quad \text { in }(u)_{\epsilon}+\stackrel{\circ}{W_{q}^{1}}\left(B_{R}\left(x_{0}\right) ; \mathbb{R}^{N}\right) .
$$

Lemma 2.1. As $\epsilon \rightarrow 0$, we have:

i) $u_{\delta} \rightarrow u$ in $W_{p}^{1}\left(B_{R}\left(x_{0}\right) ; \mathbb{R}^{N}\right)$;

ii) $\delta \int_{B_{R}\left(x_{0}\right)}\left(1+\left|\nabla u_{\delta}\right|^{2}\right)^{q / 2} \mathrm{~d} x \rightarrow 0$;

iii) $\int_{B_{R}\left(x_{0}\right)} f\left(\nabla u_{\delta}\right) \mathrm{d} x \rightarrow \int_{B_{R}\left(x_{0}\right)} f(\nabla u) \mathrm{d} x$.

Moreover, $\left\|u_{\delta}\right\|_{L^{\infty}\left(B_{R}\left(x_{0}\right), \mathbb{R}^{N}\right)}$ is uniformly bounded.

Proof. i)-iii): compare, e.g., [BF1]; the last statement follows from the convex hull property established in [BF3].

Lemma 2.2 (Caccioppoli-type inequality). For any $\eta \in C_{0}^{\infty}\left(B_{R}\left(x_{0}\right)\right)$ we have

$$
\begin{aligned}
\int_{B_{R}\left(x_{0}\right)} & \eta^{2} D^{2} f_{\delta}\left(\nabla u_{\delta}\right)\left(\partial_{\gamma} \nabla u_{\delta}, \partial_{\gamma} \nabla u_{\delta}\right) \mathrm{d} x \\
& \leq c \int_{B_{R}\left(x_{0}\right)} D^{2} f_{\delta}\left(\nabla u_{\delta}\right)\left(\nabla \eta \otimes \partial_{\gamma} u_{\delta}, \nabla \eta \otimes \partial_{\gamma} u_{\delta}\right) \mathrm{d} x
\end{aligned}
$$

Here $\gamma \in\{1, \ldots, n\}$ is arbitrary (no summation in (2.1)), $c$ is a constant independent of $\delta$, and $\otimes$ denotes the tensor product.

Proof. Compare, for example, [BF1, Lemma 3.1].

Now, let us have a closer look at inequality (2.1) for our special integrand $f$. Using (1.7) and (1.8), we see that (2.1) implies the estimate

$$
\begin{aligned}
& \delta \int_{B_{R}\left(x_{0}\right)} \eta^{2}\left(1+\left|\nabla u_{\delta}\right|^{2}\right)^{\frac{q-2}{2}}\left|\partial_{\gamma} \nabla u_{\delta}\right|^{2} \mathrm{~d} x+\int_{B_{R}\left(x_{0}\right)} \eta^{2}\left(1+\left|\tilde{\nabla} u_{\delta}\right|^{2}\right)^{\frac{p-2}{2}}\left|\partial_{\gamma} \tilde{\nabla} u_{\delta}\right|^{2} \mathrm{~d} x \\
& +\int_{B_{R}\left(x_{0}\right)} \eta^{2}\left(1+\left|\partial_{n} u_{\delta}\right|^{2}\right)^{\frac{q-2}{2}}\left|\partial_{\gamma} \partial_{n} u\right|^{2} \mathrm{~d} x \\
& \leq c\left[\delta \int_{B_{R}\left(x_{0}\right)}|\nabla \eta|^{2}\left(1+\left|\nabla u_{\delta}\right|^{2}\right)^{\frac{q-2}{2}}\left|\partial_{\gamma} u_{\delta}\right|^{2} \mathrm{~d} x\right. \\
& +\int_{B_{R}\left(x_{0}\right)}|\nabla \eta|^{2}\left(1+\left|\tilde{\nabla} u_{\delta}\right|^{2}\right)^{\frac{p-2}{2}}\left|\partial_{\gamma} u_{\delta}\right|^{2} \mathrm{~d} x \\
& \left.+\int_{B_{R}\left(x_{0}\right)}|\nabla \eta|^{2}\left(1+\left|\partial_{n} u\right|^{2}\right)^{\frac{q-2}{2}}\left|\partial_{\gamma} u_{\delta}\right|^{2} \mathrm{~d} x\right]
\end{aligned}
$$


Now, taking the sum over $\gamma$ from 1 to $n-1$ on both sides, we get

$$
\begin{aligned}
& \delta \int_{B_{R}\left(x_{0}\right)} \eta^{2} \Gamma_{\delta}^{\frac{q-2}{2}}\left|\nabla^{2} u_{\delta}\right|^{2} \mathrm{~d} x+\int_{B_{R}\left(x_{0}\right)} \eta^{2} \tilde{\Gamma}_{\delta}^{\frac{p-2}{2}}\left|\tilde{\nabla}^{2} u_{\delta}\right|^{2} \mathrm{~d} x \\
& +\int_{B_{R}\left(x_{0}\right)} \eta^{2} \Gamma_{n, \delta}^{\frac{q-2}{2}}\left|\partial_{n} \tilde{\nabla} u_{\delta}\right|^{2} \mathrm{~d} x \\
& \leq c\left[\delta \int_{B_{R}\left(x_{0}\right)}|\nabla \eta|^{2} \Gamma_{\delta}^{\frac{q-2}{2}}\left|\tilde{\nabla} u_{\delta}\right|^{2} \mathrm{~d} x\right. \\
& \left.\quad+\int_{B_{R}\left(x_{0}\right)}|\nabla \eta|^{2} \tilde{\Gamma}_{\delta}^{\frac{p-2}{2}}\left|\tilde{\nabla} u_{\delta}\right|^{2} \mathrm{~d} x+\int_{B_{R}\left(x_{0}\right)}|\nabla \eta|^{2} \Gamma_{n, \delta}^{\frac{q-2}{2}}\left|\tilde{\nabla} u_{\delta}\right|^{2} \mathrm{~d} x\right]
\end{aligned}
$$

where $\tilde{\nabla}^{2}$ has an obvious meaning, $\tilde{\Gamma}_{\delta}:=1+\left|\tilde{\nabla} u_{\delta}\right|^{2}, \Gamma_{n, \delta}:=1+\left|\partial_{n} u_{\delta}\right|^{2}$, and $\Gamma_{\delta}:=$ $1+\left|\nabla u_{\delta}\right|^{2}$.

Before proving Theorem 1.1, we recall the following auxiliary result, the proof of which can be found in $[\mathrm{GM}]$.

Proposition 2.1. Let $g: \mathbb{R}^{L} \rightarrow \mathbb{R}$ be a function of class $C^{2}$ such that for some $s \geq 2$ and a positive constant $c_{1}$ we have

$$
c_{1}\left(1+|A|^{2}\right)^{\frac{s-2}{2}}|B|^{2} \leq D^{2} g(A)(B, B) \quad \text { for all } A, B \in \mathbb{R}^{L} .
$$

Then there is another positive constant $c_{2}$, depending only on $s$ and $c_{1}$, such that

$$
\int_{0}^{1} D^{2} g(A+t B)(X, X) \mathrm{d} t \geq c_{2} D^{2} g(A)(X, X) \quad \text { for all } A, B, X \in \mathbb{R}^{L} .
$$

We proceed to a version of the Caccioppoli-type inequality involving difference quotients, which will be an essential ingredient in the proof of Theorem 1.1.

Lemma 2.3. Let $e_{\gamma}, \gamma \leq n-1$, be a fixed direction, and let $v:=\Delta_{h} u_{\delta}$ denote the difference quotient of $u_{\delta}$ in this direction. Then for any $\eta \in C_{0}^{\infty}\left(B_{R}\left(x_{0}\right)\right)$ we have

$$
\begin{aligned}
& \int_{B_{R}\left(x_{0}\right)} \eta^{2} D^{2} f_{\delta}\left(\nabla u_{\delta}\right)(\nabla v, \nabla v) \mathrm{d} x \\
& \leq c\|\nabla \eta\|_{\infty}^{2}\left[\delta \int_{\operatorname{spt} \eta} \int_{0}^{1}\left(1+|U|^{2}\right)^{\frac{q-2}{2}}|v|^{2} \mathrm{~d} t \mathrm{~d} x\right. \\
& \left.\quad+\int_{\operatorname{spt} \eta} \int_{0}^{1}\left(1+|\tilde{U}|^{2}\right)^{\frac{p-2}{2}}|v|^{2} \mathrm{~d} t \mathrm{~d} x+\int_{\operatorname{spt} \eta} \int_{0}^{1}\left(1+\left|U_{n}\right|^{2}\right)^{\frac{q-2}{2}}|v|^{2} \mathrm{~d} t \mathrm{~d} x\right] \\
& =: c\|\nabla \eta\|_{\infty}^{2}\left[\delta I_{1}+I_{2}+I_{3}\right] .
\end{aligned}
$$

Here $U:=\nabla u_{\delta}+t h \Delta_{h} \nabla u_{\delta}$.

Proof. We introduce the bilinear form $\mathcal{B}:=\int_{0}^{1} D^{2} f_{\delta}(U) \mathrm{d} t$. If we write

$$
\begin{aligned}
\mathcal{B}(X, X) & =\int_{0}^{1} D^{2} f_{\delta}\left(\nabla u_{\delta}+t h \Delta_{h} \nabla u_{\delta}\right)(X, X) \mathrm{d} t=\int_{0}^{1} D^{2} f_{\delta}(A+t B)(X, X) \mathrm{d} t \\
= & \int_{0}^{1} D^{2} g_{\delta}(A+t B)(X, X) \\
& +\int_{0}^{1} D^{2} f_{1}(\tilde{A}+t \tilde{B})(\tilde{X}, \tilde{X}) \mathrm{d} t+\int_{0}^{1} D^{2} f_{2}\left(A_{n}+t B_{n}\right)\left(X_{n}, X_{n}\right) \mathrm{d} t
\end{aligned}
$$

with $A=\nabla u_{\delta}, B=h \Delta_{h} \nabla u_{\delta}$, and $g_{\delta}(X):=\delta\left(1+|X|^{2}\right)^{q / 2}$, then, due to the ellipticity conditions imposed on $f_{1}$ and $f_{2}$, inequality (2.3) can be applied to all terms on the 
right-hand side of the above equation, leading to the estimate

$$
D^{2} f_{\delta}\left(\nabla u_{\delta}\right)(X, X) \leq \mathcal{B}(X, X) \text { for all } X \in \mathbb{R}^{n N} .
$$

Combined with (3.2) in [BF1], this gives

$$
\begin{aligned}
\int_{B_{R}\left(x_{0}\right)} & \eta^{2} D^{2} f_{\delta}\left(\nabla u_{\delta}\right)(\nabla v, \nabla v) \mathrm{d} x \\
& \leq c \int_{B_{R}\left(x_{0}\right)} \mathcal{B}(\nabla v, \nabla v) \eta^{2} \mathrm{~d} x \leq c \int_{B_{R}\left(x_{0}\right)} \eta \mathcal{B}(\nabla v, \nabla \eta \otimes v) \mathrm{d} x .
\end{aligned}
$$

Using the Cauchy-Schwarz inequality for the bilinear form $\mathcal{B}$, we get

$$
\begin{aligned}
& \int_{B_{R}\left(x_{0}\right)} \eta^{2} D^{2} f_{\delta}\left(\nabla u_{\delta}\right)(\nabla v, \nabla v) \mathrm{d} x \\
& \leq c \int_{B_{R}\left(x_{0}\right)} \mathcal{B}(\nabla \eta \otimes v, \nabla \eta \otimes v) \mathrm{d} x \\
& \leq c\left[\int_{B_{R}\left(x_{0}\right)} \int_{0}^{1} D^{2} g_{\delta}(U)(\nabla \eta \otimes v, \nabla \eta \otimes v) \mathrm{d} t \mathrm{~d} x\right. \\
& \quad+\int_{B_{R}\left(x_{0}\right)} \int_{0}^{1} D^{2} f_{1}(\tilde{U})(\tilde{\nabla} \eta \otimes v, \tilde{\nabla} \eta \otimes v) \mathrm{d} t \mathrm{~d} x \\
&\left.\quad+\int_{B_{R}\left(x_{0}\right)} \int_{0}^{1} D^{2} f_{2}\left(U_{n}\right)\left(\partial_{n} \eta v, \partial_{n} \eta v\right) \mathrm{d} t \mathrm{~d} x\right]
\end{aligned}
$$

which immediately implies the lemma, by our ellipticity assumption (1.8).

Proof of Theorem 1.1. The minimality of $u_{\delta}$ implies that

$$
\int_{B_{R}\left(x_{0}\right)} D f_{\delta}\left(\nabla u_{\delta}\right): \nabla \varphi \mathrm{d} x=0
$$

for any $\varphi \in \stackrel{\circ}{W}{ }_{q}^{1}\left(B_{R}\left(x_{0}\right) ; \mathbb{R}^{N}\right)$. As a test vector in $(2.4)$, we would like to choose $\varphi=$ $\eta^{2}\left|\tilde{\nabla} u_{\delta}\right| u_{\delta}$. The standard difference quotient procedure (see, e.g., [Mo] or [Ca]), shows only that $\tilde{\nabla} u_{\delta}$ belongs to the local Sobolev class $W_{2}^{1}$, so that the admissibility of $\varphi$ is not immediate. To overcome this problem, we fix a direction $e_{\gamma}, \gamma \leq n-1$, and, as above, let $v:=\Delta_{h} u_{\delta}$ denote the corresponding difference quotient of $u_{\delta}$. Then, using (2.4) and choosing $\varphi=\eta^{2} u_{\delta}|v|$ and $\eta \in C_{0}^{\infty}\left(B_{R}\left(x_{0}\right)\right)$, we get

$$
\begin{aligned}
& \int_{B_{R}\left(x_{0}\right)} D f_{\delta}\left(\nabla u_{\delta}\right): \nabla u_{\delta} \eta^{2}|v| \mathrm{d} x \\
& =-2 \int_{B_{R}\left(x_{0}\right)} D f_{\delta}\left(\nabla u_{\delta}\right):\left(\nabla \eta \otimes u_{\delta}\right) \eta|v| \mathrm{d} x \\
& \quad-\int_{B_{R}\left(x_{0}\right)} D f_{\delta}\left(\nabla u_{\delta}\right):\left(u_{\delta} \otimes \nabla|v|\right) \eta^{2} \mathrm{~d} x
\end{aligned}
$$

Assuming without loss of generality that $D f(0)=0$, for any matrices $X, Z$ we have $D f_{\delta}(X): Z=\int_{0}^{1} D^{2} f_{\delta}(t X)(X, Z) \mathrm{d} t$, so that, by (1.7) and (1.8), we get the estimates

$$
\begin{aligned}
& D f_{\delta}(X): X \\
& \quad \geq c\left[\delta\left(1+|X|^{2}\right)^{\frac{q-2}{2}}|X|^{2}+\left(1+|\tilde{X}|^{2}\right)^{\frac{p-2}{2}}|\tilde{X}|^{2}+\left(1+\left|X_{n}\right|^{2}\right)^{\frac{q-2}{2}}\left|X_{n}\right|^{2}\right], \\
& \left|D f_{\delta}(X)\right| \\
& \quad \leq c\left[\delta\left(1+|X|^{2}\right)^{\frac{q-2}{2}}|X|+\left(1+|\tilde{X}|^{2}\right)^{\frac{p-2}{2}}|\tilde{X}|+\left(1+\left|X_{n}\right|^{2}\right)^{\frac{q-2}{2}}\left|X_{n}\right|\right]
\end{aligned}
$$


with positive constants independent of $\epsilon$. From (2.6) we immediately deduce the fact that

$$
\begin{aligned}
& \text { l.h.s. of }(2.5) \\
& \qquad \begin{aligned}
\geq c & {\left[\delta \int_{B_{R}\left(x_{0}\right)}|v| \eta^{2} \Gamma_{\delta}^{\frac{q-2}{2}}\left|\nabla u_{\delta}\right|^{2} \mathrm{~d} x\right.} \\
& \left.+\int_{B_{R}\left(x_{0}\right)}|v| \eta^{2} \tilde{\Gamma}_{\delta}^{\frac{p-2}{2}}\left|\tilde{\nabla} u_{\delta}\right|^{2} \mathrm{~d} x+\int_{B_{R}\left(x_{0}\right)}|v| \eta^{2} \Gamma_{n, \delta}^{\frac{q-2}{2}}\left|\partial_{n} u_{\delta}\right|^{2} \mathrm{~d} x\right] .
\end{aligned}
\end{aligned}
$$

For the right-hand side of (2.5) we observe that (2.6) and the uniform $L^{\infty}$-bound of $u_{\delta}$ imply that

|the first term on the right in (2.5)|

$$
\begin{aligned}
\leq c\left[\| \nabla \eta \| _ { \infty } \left(\delta \int_{\mathrm{spt} \eta}|v| \Gamma_{\delta}^{\frac{q-2}{2}}\left|\nabla u_{\delta}\right| \mathrm{d} x\right.\right. & \left.+\int_{\operatorname{spt} \eta}|v| \tilde{\Gamma}_{\delta}^{\frac{p-2}{2}}\left|\tilde{\nabla} u_{\delta}\right| \mathrm{d} x\right) \\
& \left.+\int_{\operatorname{spt} \eta} \eta|\nabla \eta||v| \Gamma_{n, \delta}^{\frac{q-2}{2}}\left|\partial_{n} u_{\delta}\right| \mathrm{d} x\right] \\
= & : c\left[\|\nabla \eta\|_{\infty}\left(T_{1}+T_{2}\right)+T_{3}\right] .
\end{aligned}
$$

Using Hölder's inequality, the elementary properties of difference quotients, and Lemma 2.1 , ii), we see that

$$
T_{1} \leq c \delta \int_{B_{R}\left(x_{0}\right)} \Gamma_{\delta}^{\frac{q}{2}} \mathrm{~d} x \rightarrow 0 \quad \text { as } \epsilon \rightarrow 0,
$$

whereas

$$
T_{2} \leq c \int_{B_{R}\left(x_{0}\right)} \tilde{\Gamma}_{\delta}^{\frac{p}{2}} \mathrm{~d} x \leq c
$$

by Lemma 2.2. Next, for any $\tau \in(0,1)$ we have

$$
\begin{aligned}
T_{3} & \leq \int_{\operatorname{spt} \eta}\left[\tau \eta^{2}\left|\partial_{n} u_{\delta}\right|^{2}+\frac{1}{\tau}\|\nabla \eta\|_{\infty}^{2}\right]|v| \Gamma_{n, \delta}^{\frac{q-2}{2}} \mathrm{~d} x \\
& \leq \tau \int_{\operatorname{spt} \eta} \eta^{2}\left|v\left\|\left.\partial_{n} u_{\delta}\right|^{2} \Gamma_{n, \delta}^{\frac{q-2}{2}} \mathrm{~d} x+\frac{c}{\tau}\right\| \nabla \eta \|_{\infty}^{2} \int_{\operatorname{spt} \eta}\left\{\Gamma_{n, \delta}^{\frac{q}{2}}+|v|^{\frac{q}{2}}\right\} \mathrm{d} x .\right.
\end{aligned}
$$

Since it is assumed that $q \leq 2 p$, we conclude that

$$
\int_{\operatorname{spt} \eta}|v|^{\frac{q}{2}} \mathrm{~d} x
$$

is bounded in terms of $\int_{B_{R}\left(x_{0}\right)}\left|\tilde{\nabla} u_{\delta}\right|^{p} \mathrm{~d} x$. Moreover, if $\tau$ is sufficiently small, then the first integral on the right in (2.9) can be absorbed in the last integral on the right in (2.8) with the following result (without loss of generality we assume that $\|\nabla \eta\|_{\infty} \geq 1$ ):

$$
\begin{aligned}
& \delta \int_{B_{R}\left(x_{0}\right)}|v| \eta^{2} \Gamma_{\delta}^{\frac{q-2}{2}}\left|\nabla u_{\delta}\right|^{2} \mathrm{~d} x \\
& +\int_{B_{R}\left(x_{0}\right)}|v| \eta^{2} \tilde{\Gamma}_{\delta}^{\frac{p-2}{2}}\left|\tilde{\nabla} u_{\delta}\right|^{2} \mathrm{~d} x+\int_{B_{R}\left(x_{0}\right)}|v| \eta^{2} \Gamma_{n, \delta}^{\frac{q-2}{2}}\left|\partial_{n} u_{\delta}\right|^{2} \mathrm{~d} x \\
& \quad \leq c\|\nabla \eta\|_{\infty}^{2}+c\left|\int_{B_{R}\left(x_{0}\right)} D f_{\delta}\left(\nabla u_{\delta}\right):\left(u_{\delta} \otimes \nabla|v|\right) \eta^{2} \mathrm{~d} x\right|
\end{aligned}
$$

We pass to the remaining integral on the right-hand side of (2.10): we observe that

$$
D f_{\delta}(X): Y=\delta \frac{q}{2}\left(1+|X|^{2}\right)^{\frac{q-2}{2}} X: Y+D f_{1}(\tilde{X}): \tilde{Y}+D f_{2}\left(X_{n}\right) \cdot Y_{n},
$$


which implies, by using the uniform boundedness of $u_{\delta}$, that

$$
\begin{aligned}
& \left|D f_{\delta}\left(\nabla u_{\delta}\right):\left(\nabla|v| \otimes u_{\delta}\right)\right| \\
& \leq c\left[\delta \Gamma_{\delta}^{\frac{q-2}{2}}\left|\nabla u_{\delta}\right||\nabla v|+\tilde{\Gamma}_{\delta}^{\frac{p-2}{2}}\left|\tilde{\nabla} u_{\delta}\right||\tilde{\nabla} v|+\Gamma_{n, \delta}^{\frac{q-2}{2}}\left|\partial_{n} u_{\delta}\right|\left|\partial_{n} v\right|\right] \\
& \leq c\left[\delta \tau \Gamma_{\delta}^{\frac{q-2}{2}}|\nabla v|^{2}+\delta \frac{1}{\tau} \Gamma_{\delta}^{\frac{q-2}{2}}\left|\nabla u_{\delta}\right|^{2}+\tau \tilde{\Gamma}_{\delta}^{\frac{p-2}{2}}|\tilde{\nabla} v|^{2}+\frac{1}{\tau} \tilde{\Gamma}_{\delta}^{\frac{p}{2}}\right. \\
& \left.\quad+\tau \Gamma_{n, \delta}^{\frac{q-2}{2}}\left|\partial_{n} v\right|^{2}+\frac{1}{\tau} \Gamma_{n, \delta}^{\frac{q-2}{2}}\left|\partial_{n} u_{\delta}\right|^{2}\right]
\end{aligned}
$$

for any $0<\tau<1$. This gives the following upper bound for the integral under consideration:

$$
\begin{array}{r}
c\left[\delta \tau \int_{B_{R}\left(x_{0}\right)} \eta^{2} \Gamma_{\delta}^{\frac{q-2}{2}}|\nabla v|^{2} \mathrm{~d} x+\tau \int_{B_{R}\left(x_{0}\right)} \tilde{\Gamma}_{\delta}^{\frac{p-2}{2}} \eta^{2}|\tilde{\nabla} v|^{2} \mathrm{~d} x\right. \\
+\tau \int_{B_{R}\left(x_{0}\right)} \eta^{2} \Gamma_{n, \delta}^{\frac{q-2}{2}}\left|\partial_{n} v\right|^{2} \mathrm{~d} x+\frac{1}{\tau} \int_{B_{R}\left(x_{0}\right)} \eta^{2} \tilde{\Gamma}_{\delta}^{\frac{p}{2}} \mathrm{~d} x \\
\left.+\frac{1}{\tau} \delta \int_{B_{R}\left(x_{0}\right)} \eta^{2} \Gamma_{\delta}^{\frac{q}{2}} \mathrm{~d} x+\frac{1}{\tau} \int_{B_{R}\left(x_{0}\right)} \Gamma_{n, \delta}^{\frac{q}{2}} \mathrm{~d} x\right],
\end{array}
$$

where all the quantities multiplied by $\frac{1}{\tau}$ stay bounded uniformly with respect to $\epsilon$ (recall that $\delta=\delta(\epsilon)$ ). In order to continue, we look at the sum of the first three terms of (2.12), observing that

$$
\begin{gathered}
\int_{B_{R}\left(x_{0}\right)} \eta^{2}\left[\delta \Gamma_{\delta}^{\frac{q-2}{2}}|\nabla v|^{2}+\tilde{\Gamma}_{\delta}^{\frac{p-2}{2}}|\tilde{\nabla} v|^{2}+\Gamma_{n, \delta}^{\frac{q-2}{2}}\left|\partial_{n} v\right|^{2}\right] \mathrm{d} x \\
\leq c \int_{B_{R}\left(x_{0}\right)} \eta^{2} D^{2} f_{\delta}\left(\nabla u_{\delta}\right)(\nabla v, \nabla v) \mathrm{d} x .
\end{gathered}
$$

Here the right-hand side can be estimated with the help of Lemma 2.3; i.e. (with the notation of Lemma 2.3), inequality (2.10) implies that, for any $0<\tau<1$,

$$
\begin{aligned}
& \delta \int_{B_{R}\left(x_{0}\right)}|v| \eta^{2} \Gamma_{\delta}^{\frac{q-2}{2}}\left|\nabla u_{\delta}\right|^{2} \mathrm{~d} x \\
& +\int_{B_{R}\left(x_{0}\right)}|v| \eta^{2} \tilde{\Gamma}_{\delta}^{\frac{p-2}{2}}\left|\tilde{\nabla} u_{\delta}\right|^{2} \mathrm{~d} x+\int_{B_{R}\left(x_{0}\right)}|v| \eta^{2} \Gamma_{n, \delta}^{\frac{q-2}{2}}\left|\partial_{n} u_{\delta}\right|^{2} \mathrm{~d} x \\
& \quad \leq c\|\nabla \eta\|_{\infty}^{2}+c \tau\|\nabla \eta\|_{\infty}^{2}\left[\delta I_{1}+I_{2}+I_{3}\right]+\frac{c}{\tau} .
\end{aligned}
$$

We have

$$
\begin{aligned}
I_{1} & \leq \int_{\operatorname{spt} \eta}|v|^{q} \mathrm{~d} x+\int_{\operatorname{spt} \eta} \int_{0}^{1}\left(1+|U|^{2}\right)^{\frac{q}{2}} \mathrm{~d} t \mathrm{~d} x \\
& \leq c \int_{B_{R}\left(x_{0}\right)}\left|\nabla u_{\delta}\right|^{q} \mathrm{~d} x+c \int_{\operatorname{spt} \eta} \int_{0}^{1}\left(\left|(1-t) \nabla u_{\delta}(x)+t \nabla u_{\delta}\left(x+h e_{\gamma}\right)\right|^{2}+1\right)^{\frac{q}{2}} \mathrm{~d} t \mathrm{~d} x,
\end{aligned}
$$

and if from now on we assume that $\operatorname{spt} \eta \subset B_{R / 2}\left(x_{0}\right)$, then of course

$$
I_{1} \leq c \int_{B_{R}\left(x_{0}\right)} \Gamma_{\delta}^{\frac{q}{2}} \mathrm{~d} x
$$

uniformly in $\varepsilon$ (provided $|h| \ll 1$ ). Since we know that $\delta I_{1} \rightarrow 0$ as $\epsilon \rightarrow 0$, the quantity $\delta I_{1}$ is uniformly bounded for all small $\epsilon$ and $|h|$. Since we also know that $\int_{B_{R}\left(x_{0}\right)} \tilde{\Gamma}_{\delta}^{p / 2} \mathrm{~d} x \leq c$, the same argument applies to $I_{2}$. To handle $I_{3}$, observe that, in the limit as $h \rightarrow 0$,

$$
I_{3} \leq \int_{\operatorname{spt} \eta} \int_{0}^{1}\left(1+\left|U_{n}\right|^{2}\right)^{\frac{q-2}{2}}|v|^{2} \mathrm{~d} t \mathrm{~d} x \rightarrow \int_{\operatorname{spt} \eta} \Gamma_{n, \delta}^{\frac{q-2}{2}}\left|\partial_{\gamma} u_{\delta}\right|^{2} \mathrm{~d} x .
$$


To prove (2.14), we note that, by [Gi1, Theorem 3.1, p. 159], there exists an exponent $s>q$ (depending on $\epsilon$ ) such that $\nabla u_{\delta} \in L_{\text {loc }}^{s}\left(B_{R}\left(x_{0}\right) ; \mathbb{R}^{n N}\right)$. This implies that, as $h \rightarrow 0$,

$$
\partial_{n} u_{\delta}\left(x+h e_{\gamma}\right) \rightarrow \partial_{n} u_{\delta}, \quad v \rightarrow \partial_{\gamma} u_{\delta}
$$

in $L_{\text {loc }}^{s}\left(B_{R}\left(x_{0}\right) ; \mathbb{R}^{N}\right)$ and a.e. Clearly, as $h \rightarrow 0$,

$$
\zeta_{h}:=\int_{0}^{1}\left(1+\left|U_{n}\right|^{2}\right)^{\frac{q-2}{2}} \mathrm{~d} t|v|^{2} \rightarrow \Gamma_{n, \delta}^{\frac{q-2}{2}}\left|\partial_{\gamma} u_{\delta}\right|^{2}
$$

a.e., and we get the equi-integrability of the $\zeta_{h}$ by using the local $L^{s}$-convergences. Then (2.14) follows from the Vitali convergence theorem. Returning to (2.13), using (2.14) and the foregoing estimates, and applying Fatou's lemma on the left-hand side of (2.13), in the limit as $h \rightarrow 0$ we find

$$
\begin{aligned}
& \delta \int_{B_{s}(\bar{x})}\left|\partial_{\gamma} u_{\delta}\right| \Gamma_{\delta}^{\frac{q-2}{2}}\left|\nabla u_{\delta}\right|^{2} \mathrm{~d} x \\
& +\int_{B_{s}(\bar{x})}\left|\partial_{\gamma} u_{\delta}\right| \tilde{\Gamma}_{\delta}^{\frac{p-2}{2}}\left|\tilde{\nabla} u_{\delta}\right|^{2} \mathrm{~d} x+\int_{B_{s}(\bar{x})}\left|\partial_{\gamma} u_{\delta}\right| \Gamma_{n, \delta}^{\frac{q-2}{2}}\left|\partial_{n} u_{\delta}\right|^{2} \mathrm{~d} x \\
& \quad \leq c(t-s)^{-2}+\frac{c}{\tau}+c \tau(t-s)^{-2} \int_{B_{t}(\bar{x})} \Gamma_{n, \delta}^{\frac{q-2}{2}}\left|\partial_{\gamma} u_{\delta}\right|^{2} \mathrm{~d} x
\end{aligned}
$$

where $\eta \in C_{0}^{\infty}\left(B_{t}(\bar{x})\right)\left(0<s<t<T, B_{T}(\bar{x}) \Subset B_{R / 2}\left(x_{0}\right)\right)$ is such that $0 \leq \eta \leq 1, \eta \equiv 1$ on $B_{s}(\bar{x})$, and $|\nabla \eta| \leq c /(t-s)$. The last integral in (2.15) can be estimated as follows. By Young's inequality, we have

$$
\begin{aligned}
\int_{B_{t}(\bar{x})} & \Gamma_{n, \delta}^{\frac{q-2}{2}}\left|\partial_{\gamma} u_{\delta}\right|^{2} \mathrm{~d} x \\
& \leq c \int_{B_{t}(\bar{x})}\left|\partial_{n} u_{\delta}\right|^{q-2}\left|\partial_{\gamma} u_{\delta}\right|^{2} \mathrm{~d} x+c \int_{B_{t}(\bar{x})}\left|\partial_{\gamma} u_{\delta}\right|^{2} \mathrm{~d} x \\
& \leq c \int_{B_{t}(\bar{x})}\left|\partial_{\gamma} u_{\delta}\right|\left|\partial_{n} u_{\delta}\right|^{q} \mathrm{~d} x+c \int_{B_{t}(\bar{x})}\left(\left|\partial_{\gamma} u_{\delta}\right|^{\frac{q}{2}+1}+\left|\partial_{\gamma} u_{\delta}\right|^{2} \mid\right) \mathrm{d} x .
\end{aligned}
$$

Moreover, since $q \leq 2 p$,

$$
\begin{aligned}
\left|\partial_{\gamma} u_{\delta}\right|^{\frac{q}{2}+1} & \leq \tilde{\Gamma}_{\delta}^{\frac{p-2}{2}}\left|\partial_{\gamma} u_{\delta}\right|^{\frac{q}{2}+1-p+2} \leq \tilde{\Gamma}_{\delta}^{\frac{p-2}{2}}\left|\tilde{\nabla} u_{\delta}\right|^{2}\left|\partial_{\gamma} u_{\delta}\right|^{\frac{q}{2}-p+1} \\
& \leq \tilde{\Gamma}_{\delta}^{\frac{p-2}{2}}\left|\tilde{\nabla} u_{\delta}\right|^{2}\left[\left|\partial_{\gamma} u_{\delta}\right|+1\right]
\end{aligned}
$$

provided $q / 2-p+1 \geq 0$. If this is not the case, i.e., $q \leq 2 p-2$, then

$$
\begin{aligned}
\left|\partial_{\gamma} u_{\delta}\right|^{\frac{q}{2}+1} & \leq\left|\tilde{\nabla} u_{\delta}\right|^{\frac{q}{2}+1} \leq\left|\tilde{\nabla} u_{\delta}\right|^{2} \tilde{\Gamma}_{\delta}^{\frac{q-2}{4}} \leq\left|\tilde{\nabla} u_{\delta}\right|^{2} \tilde{\Gamma}_{\delta}^{\frac{p-2}{2}} \\
& \leq \tilde{\Gamma}_{\delta}^{\frac{p-2}{2}}\left|\tilde{\nabla} u_{\delta}\right|^{2}\left[\left|\partial_{\gamma} u_{\delta}\right|+1\right]
\end{aligned}
$$

Collecting all this, we get

$$
\begin{aligned}
& \int_{B_{t}(\bar{x})} \Gamma_{n, \delta}^{\frac{q-2}{2}}\left|\partial_{\gamma} u_{\delta}\right|^{2} \mathrm{~d} x \\
& \quad \leq c\left[\int_{B_{t}(\bar{x})}\left|\partial_{\gamma} u_{\delta}\right|\left|\partial_{n} u_{\delta}\right|^{q} \mathrm{~d} x+\int_{B_{t}(\bar{x})} \tilde{\Gamma}_{\delta}^{\frac{p-2}{2}}\left|\tilde{\nabla} u_{\delta}\right|^{2}\left|\partial_{\gamma} u_{\delta}\right| \mathrm{d} x+1\right] .
\end{aligned}
$$


Inserting this estimate in (2.15) and neglecting the first term on the left in (2.15) we see that

$$
\begin{aligned}
& h(s):=\int_{B_{s}(\bar{x})}\left|\partial_{\gamma} u_{\delta}\right| \tilde{\Gamma}_{\delta}^{\frac{p-2}{2}}\left|\tilde{\nabla} u_{\delta}\right|^{2} \mathrm{~d} x+\int_{B_{s}(\bar{x})}\left|\partial_{\gamma} u_{\delta}\right| \Gamma_{n, \delta}^{\frac{q-2}{2}}\left|\partial_{n} u_{\delta}\right|^{2} \mathrm{~d} x \\
& \leq c(t-s)^{-2}+\frac{c}{\tau}+c \tau(t-s)^{-2} \\
& \quad+c \tau(t-s)^{-2}\left[\int_{B_{t}(\bar{x})} \tilde{\Gamma}_{\delta}^{\frac{p-2}{2}}\left|\tilde{\nabla} u_{\delta}\right|^{2}\left|\partial_{\gamma} u_{\delta}\right| \mathrm{d} x+\int_{B_{t}(\bar{x})}\left|\partial_{\gamma} u_{\delta}\right| \Gamma_{n, \delta}^{\frac{q-2}{2}}\left|\partial_{n} u_{\delta}\right|^{2} \mathrm{~d} x\right]
\end{aligned}
$$

i.e., with the choice $\tau=(t-s)^{2} /(2 c)$,

$$
h(s) \leq c(t-s)^{-2}+c+\frac{1}{2} h(t)
$$

for any $s, t$ as above. Finally, we use [Gi1, Lemma 3.1, p. 161] to show that the estimate

$$
\int_{B_{s}(\bar{x})}\left|\partial_{\gamma} u_{\delta}\right| \tilde{\Gamma}_{\delta}^{\frac{p-2}{2}}\left|\tilde{\nabla} u_{\delta}\right|^{2} \mathrm{~d} x+\int_{B_{s}(\bar{x})}\left|\partial_{\gamma} u_{\delta}\right| \Gamma_{n, \delta}^{\frac{q-2}{2}}\left|\partial_{n} u_{\delta}\right|^{2} \mathrm{~d} x \leq c\left[(T-s)^{-2}+1\right]
$$

is valid for all $s<T$ with a constant $c$ independent of $\epsilon$. Recalling that $\gamma \leq n-1$ we get the uniform bound

$$
\int_{B_{R / 4}\left(x_{0}\right)}\left|\tilde{\nabla} u_{\delta}\right|^{p+1} \mathrm{~d} x+\int_{B_{R / 4}\left(x_{0}\right)}\left|\tilde{\nabla} u_{\delta}\right|\left|\partial_{n} u_{\delta}\right|^{q} \mathrm{~d} x \leq c(R) .
$$

Since by Lemma 2.1 we already know that $u_{\delta} \rightarrow u$ in $W_{p}^{1}\left(B_{R}\left(x_{0}\right) ; \mathbb{R}^{N}\right)$ as $\epsilon \rightarrow 0$, the first claim of Theorem 1.1 follows.

The second statement is obtained by a similar calculation, with the replacement of the function $v$ by the difference quotient of $u$ in the $n$th coordinate direction. We also refer to the last section, where this calculation is carried out for the scalar case (avoiding the difference-quotient technique).

The statement of the corollary is an immediate consequence of the Sobolev embedding theorem.

\section{§3. Higher InTEGRABiLity IN THE SCALAR CASE:}

THE FIRST STEP IN THE PROOF OF THEOREM 1.2

In this section we prove that $\tilde{\nabla} u \in L_{\text {loc }}^{s}\left(\Omega ; \mathbb{R}^{n-1}\right)$ for any finite exponent $s$; in particular, actually $u$ is in the space $W_{q, \text { loc }}^{1}(\Omega)$. We use the notation introduced in $\S 2$. The results of [LU] (see the discussion in Remark 2.3 of [BF4]) imply that $u_{\delta}$ is in the space $W_{t, \text { loc }}^{2}\left(B_{R}\left(x_{0}\right)\right)$ for any $t<\infty$; therefore we may test the differentiated Euler equation, valid for $u_{\delta}$, with the function $\eta^{2} \partial_{\gamma} u_{\delta} \tilde{\Gamma}_{\delta}^{\beta / 2}$, where $\beta \geq 0, \eta \in C_{0}^{\infty}\left(B_{R}\left(x_{0}\right)\right)$, and $\gamma$ runs from 1 to $n-1$. Since we consider the scalar case, it is easy to check that

$$
0 \leq \int_{B_{R}\left(x_{0}\right)} D^{2} f_{\delta}\left(\nabla u_{\delta}\right)\left(\partial_{\gamma} \nabla u_{\delta}, \eta^{2} \partial_{\gamma} u_{\delta} \nabla \tilde{\Gamma}_{\delta}^{\frac{\beta}{2}}\right) \mathrm{d} x
$$

(from now on summation over $\gamma$ from 1 to $n-1$ is assumed). In fact, this is the only point where the condition $N=1$ is needed. Thus, (2.1) is replaced by the inequality

$$
\begin{aligned}
& \int_{B_{R}\left(x_{0}\right)} \tilde{\Gamma}_{\delta}^{\frac{\beta}{2}} \eta^{2} D^{2} f_{\delta}\left(\nabla u_{\delta}\right)\left(\partial_{\gamma} \nabla u_{\delta}, \partial_{\gamma} \nabla u_{\delta}\right) \mathrm{d} x \\
& \quad \leq c \int_{B_{R}\left(x_{0}\right)} \tilde{\Gamma}_{\delta}^{\frac{\beta}{2}} D^{2} f_{\delta}\left(\nabla u_{\delta}\right)\left(\nabla \eta \partial_{\gamma} u_{\delta}, \nabla \eta \partial_{\gamma} u_{\delta}\right) \mathrm{d} x
\end{aligned}
$$


which means that we get $(2.2)$ with the factor $\tilde{\Gamma}_{\delta}^{\beta / 2}$ on both sides:

$$
\begin{aligned}
& \delta \int_{B_{R}\left(x_{0}\right)} \tilde{\Gamma}^{\frac{\beta}{2}} \eta^{2} \Gamma_{\delta}^{\frac{q-2}{2}}\left|\nabla^{2} u_{\delta}\right|^{2} \mathrm{~d} x+\int_{B_{R}\left(x_{0}\right)} \tilde{\Gamma}^{\frac{\beta}{2}} \eta^{2} \tilde{\Gamma}_{\delta}^{\frac{p-2}{2}}\left|\tilde{\nabla}^{2} u_{\delta}\right|^{2} \mathrm{~d} x \\
& +\int_{B_{R}\left(x_{0}\right)} \tilde{\Gamma}^{\frac{\beta}{2}} \eta^{2} \Gamma_{n, \delta}^{\frac{q-2}{2}}\left|\partial_{n} \tilde{\nabla} u_{\delta}\right|^{2} \mathrm{~d} x \\
& \leq c\left[\delta \int_{B_{R}\left(x_{0}\right)} \tilde{\Gamma}^{\frac{\beta}{2}}|\nabla \eta|^{2} \Gamma_{\delta}^{\frac{q-2}{2}}\left|\nabla u_{\delta}\right|^{2} \mathrm{~d} x\right. \\
& \left.\quad+\int_{B_{R}\left(x_{0}\right)} \tilde{\Gamma}^{\frac{\beta}{2}}|\nabla \eta|^{2} \tilde{\Gamma}_{\delta}^{\frac{p-2}{2}}\left|\tilde{\nabla} u_{\delta}\right|^{2} \mathrm{~d} x+\int_{B_{R}\left(x_{0}\right)} \tilde{\Gamma}^{\frac{\beta}{2}}|\nabla \eta|^{2} \Gamma_{n, \delta}^{\frac{q-2}{2}}\left|\tilde{\nabla} u_{\delta}\right|^{2} \mathrm{~d} x\right] .
\end{aligned}
$$

We apply (2.4) with $\varphi:=\eta^{2} u_{\delta} \tilde{\Gamma}_{\delta}^{(1+\alpha) / 2}$ as an admissible test function; here $\alpha \geq 0$ is some number to be specified below. As a result, we get (2.5) with $|v|$ replaced by $\tilde{\Gamma}_{\delta}^{(1+\alpha) / 2}$ :

$$
\begin{aligned}
\int_{B_{R}\left(x_{0}\right)} D f_{\delta}\left(\nabla u_{\delta}\right) \cdot \nabla u_{\delta} \eta^{2} \tilde{\Gamma}_{\delta}^{\frac{1+\alpha}{2}} \mathrm{~d} x \\
=-2 \int_{B_{R}\left(x_{0}\right)} D f_{\delta}\left(\nabla u_{\delta}\right) \cdot \nabla \eta u_{\delta} \eta \tilde{\Gamma}_{\delta}^{\frac{1+\alpha}{2}} \mathrm{~d} x \\
\quad-\int_{B_{R}\left(x_{0}\right)} D f_{\delta}\left(\nabla u_{\delta}\right) \cdot u_{\delta} \nabla \tilde{\Gamma}_{\delta}^{\frac{1+\alpha}{2}} \eta^{2} \mathrm{~d} x
\end{aligned}
$$

We observe (cf. (2.8))

(3.1) the left-hand side of $\left(2.5_{\alpha}\right)$

$$
\begin{aligned}
\geq c\left[\delta \int_{B_{R}\left(x_{0}\right)} \Gamma_{\delta}^{\frac{q-2}{2}} \tilde{\Gamma}_{\delta}^{\frac{1+\alpha}{2}} \eta^{2}\left|\nabla u_{\delta}\right|^{2} \mathrm{~d} x\right. & +\int_{B_{R}\left(x_{0}\right)} \tilde{\Gamma}_{\delta}^{\frac{p-2}{2}} \tilde{\Gamma}_{\delta}^{\frac{1+\alpha}{2}} \eta^{2}\left|\tilde{\nabla} u_{\delta}\right|^{2} \mathrm{~d} x \\
& \left.+\int_{B_{R}\left(x_{0}\right)} \tilde{\Gamma}_{\delta}^{\frac{1+\alpha}{2}} \Gamma_{n, \delta}^{\frac{q-2}{2}} \eta^{2}\left|\partial_{n} u_{\delta}\right|^{2} \mathrm{~d} x\right] .
\end{aligned}
$$

Next, we estimate the first term on the right in $\left(2.5_{\alpha}\right)$ (compare the inequality after (2.10)):

$$
\begin{aligned}
& \left|2 \int_{B_{R}\left(x_{0}\right)} D f_{\delta}\left(\nabla u_{\delta}\right) \cdot \nabla \eta u_{\delta} \eta \tilde{\Gamma}_{\delta}^{\frac{1+\alpha}{2}} \mathrm{~d} x\right| \\
& \leq c\|\nabla \eta\|_{\infty}\left[\delta \int_{\operatorname{spt} \eta} \tilde{\Gamma}_{\delta}^{\frac{1+\alpha}{2}} \Gamma_{\delta}^{\frac{q-2}{2}}\left|\nabla u_{\delta}\right| \mathrm{d} x+\int_{\operatorname{spt} \eta} \tilde{\Gamma}_{\delta}^{\frac{1+\alpha}{2}} \tilde{\Gamma}_{\delta}^{\frac{p-2}{2}}\left|\tilde{\nabla} u_{\delta}\right| \mathrm{d} x\right. \\
& \left.+\int_{\operatorname{spt} \eta} \tilde{\Gamma}_{\delta}^{\frac{1+\alpha}{2}} \Gamma_{n, \delta}^{\frac{q-2}{2}}\left|\partial_{n} u_{\delta}\right| \mathrm{d} x\right] \\
& \leq c\|\nabla \eta\|_{\infty}\left[\delta \int_{\operatorname{spt} \eta} \Gamma_{\delta}^{\frac{q-2}{2}} \tilde{\Gamma}_{\delta}^{\frac{1+\alpha}{2}}\left|\nabla u_{\delta}\right| \mathrm{d} x+\int_{\operatorname{spt} \eta} \tilde{\Gamma}_{\delta}^{\frac{p+\alpha}{2}} \mathrm{~d} x+\int_{\operatorname{spt} \eta} \tilde{\Gamma}_{\delta}^{\frac{1+\alpha}{2}} \Gamma_{n, \delta}^{\frac{q-1}{2}} \mathrm{~d} x\right] \\
& \leq c\|\nabla \eta\|_{\infty}\left[\tau \delta \int_{\operatorname{spt} \eta} \Gamma_{\delta}^{\frac{q-2}{2}} \tilde{\Gamma}_{\delta}^{\frac{1+\alpha}{2}}\left|\nabla u_{\delta}\right|^{2} \mathrm{~d} x+c(\tau) \delta \int_{\operatorname{spt} \eta} \Gamma_{\delta}^{\frac{q-2}{2}} \tilde{\Gamma}_{\delta}^{\frac{1+\alpha}{2}} \mathrm{~d} x\right. \\
& +\tau \int_{\operatorname{spt} \eta} \tilde{\Gamma}_{\delta}^{\frac{p+\alpha+1}{2}} \mathrm{~d} x+c(\tau) \int_{\operatorname{spt} \eta} \tilde{\Gamma}_{\delta}^{\frac{p+\alpha-1}{2}} \mathrm{~d} x \\
& \left.+\tau \int_{\operatorname{spt} \eta} \tilde{\Gamma}_{\delta}^{\frac{1+\alpha}{2}} \Gamma_{n, \delta}^{\frac{q}{2}} \mathrm{~d} x+c(\tau) \int_{\operatorname{spt} \eta} \tilde{\Gamma}_{\delta}^{\frac{1+\alpha}{2}} \mathrm{~d} x\right],
\end{aligned}
$$


where $0<\tau<1$ is arbitrary. In order to handle the second term on the right in $\left(2.5_{\alpha}\right)$, we recall $(2.11)$, obtaining

$$
\begin{aligned}
& \left|D f_{\delta}\left(\nabla u_{\delta}\right) \cdot \nabla \tilde{\Gamma}_{\delta}^{\frac{\alpha+1}{2}}\right| \\
& \quad \leq c \frac{\alpha+1}{2} \tilde{\Gamma}_{\delta}^{\frac{\alpha-1}{2}}\left[\delta \Gamma_{\delta}^{\frac{q-2}{2}}\left|\nabla u_{\delta}\right|\left|\nabla \tilde{\Gamma}_{\delta}\right|+\tilde{\Gamma}_{\delta}^{\frac{p-2}{2}}\left|\tilde{\nabla} u_{\delta}\right|\left|\tilde{\nabla}_{\Gamma_{\delta}}\right|+\Gamma_{n, \delta}^{\frac{q-2}{2}}\left|\partial_{n} u_{\delta}\right|\left|\partial_{n} \tilde{\Gamma}_{\delta}\right|\right] \\
& \quad \leq c(\alpha)\left[\delta \tilde{\Gamma}_{\delta}^{\frac{\alpha}{2}} \Gamma_{\delta}^{\frac{q-1}{2}}\left|\nabla^{2} u_{\delta}\right|+\tilde{\Gamma}_{\delta}^{\frac{p+\alpha-1}{2}}\left|\tilde{\nabla}^{2} u_{\delta}\right|+\tilde{\Gamma}_{\delta}^{\frac{\alpha}{2}} \Gamma_{n, \delta}^{\frac{q-1}{2}}\left|\partial_{n} \tilde{\nabla} u_{\delta}\right|\right] .
\end{aligned}
$$

We have

$$
\begin{gathered}
\tilde{\Gamma}_{\delta}^{\frac{p+\alpha-1}{2}}\left|\tilde{\nabla}^{2} u_{\delta}\right| \leq \tilde{\Gamma}_{\delta}^{\frac{\beta}{2}} \tilde{\Gamma}_{\delta}^{\frac{p-2}{2}}\left|\tilde{\nabla}^{2} u_{\delta}\right|^{2}+\tilde{\Gamma}_{\delta}^{-\frac{\beta}{2}+\frac{p+2 \alpha}{2}} \\
\delta \tilde{\Gamma}_{\delta}^{\frac{\alpha}{2}} \Gamma_{\delta}^{\frac{q-1}{2}}\left|\nabla^{2} u_{\delta}\right| \leq \delta \Gamma_{\delta}^{\frac{q-2}{2}} \tilde{\Gamma}_{\delta}^{\frac{\beta}{2}}\left|\nabla^{2} u_{\delta}\right|^{2}+\delta \Gamma_{\delta}^{\frac{q}{2}} \tilde{\Gamma}_{\delta}^{\frac{2 \alpha-\beta}{2}}
\end{gathered}
$$

as well as

$$
\begin{gathered}
\tilde{\Gamma}_{\delta}^{\frac{\alpha}{2}} \Gamma_{n, \delta}^{\frac{q-1}{2}}\left|\partial_{n} \tilde{\nabla} u_{\delta}\right|=\tilde{\Gamma}_{\delta}^{\frac{\beta}{4}} \Gamma_{n \delta}^{\frac{q-2}{4}}\left|\partial_{n} \tilde{\nabla} u_{\delta}\right| \tilde{\Gamma}_{\delta}^{-\frac{\beta}{4}+\frac{\alpha}{2}} \Gamma_{n, \delta}^{\frac{q}{4}} \\
\quad \leq \tilde{\Gamma}_{\delta}^{\frac{\beta}{2}} \Gamma_{n, \delta}^{\frac{q-2}{2}}\left|\partial_{n} \tilde{\nabla} u_{\delta}\right|^{2}+\Gamma_{n, \delta}^{\frac{q}{2}} \tilde{\Gamma}_{\delta}^{\frac{2 \alpha-\beta}{2}}
\end{gathered}
$$

Inserting these inequalities in (3.3), we get

$$
\begin{aligned}
& \left|\int_{B_{R}\left(x_{0}\right)} D f_{\delta}\left(\nabla u_{\delta}\right) \cdot \nabla \Gamma_{\delta}^{\frac{\alpha+1}{2}} u_{\delta} \eta^{2} \mathrm{~d} x\right| \\
& \leq c(\alpha)\left[\delta \int_{B_{R}\left(x_{0}\right)} \eta^{2} \tilde{\Gamma}_{\delta}^{\frac{\beta}{2}} \Gamma_{\delta}^{\frac{q-2}{2}}\left|\nabla^{2} u_{\delta}\right|^{2} \mathrm{~d} x+\int_{B_{R}\left(x_{0}\right)} \eta^{2} \tilde{\Gamma}_{\delta}^{\frac{\beta}{2}} \tilde{\Gamma}_{\delta}^{\frac{p-2}{2}}\left|\tilde{\nabla}^{2} u_{\delta}\right|^{2} \mathrm{~d} x\right. \\
& \left.+\int_{B_{R}\left(x_{0}\right)} \eta^{2} \tilde{\Gamma}_{\delta}^{\frac{\beta}{2}} \Gamma_{n, \delta}^{\frac{q-2}{2}}\left|\partial_{n} \tilde{\nabla} u_{\delta}\right|^{2} \mathrm{~d} x\right] \\
& +c(\alpha)\left[\delta \int_{B_{R}\left(x_{0}\right)} \eta^{2} \Gamma_{\delta}^{\frac{q}{2}} \tilde{\Gamma}_{\delta}^{\frac{2 \alpha-\beta}{2}} \mathrm{~d} x\right. \\
& \left.\quad+\int_{B_{R}\left(x_{0}\right)} \eta^{2} \tilde{\Gamma}_{\delta}^{\frac{p+2 \alpha-\beta}{2}} \mathrm{~d} x+\int_{B_{R}\left(x_{0}\right)} \eta^{2} \Gamma_{n, \delta}^{\frac{q}{2}} \tilde{\Gamma}_{\delta}^{\frac{2 \alpha-\beta}{2}} \mathrm{~d} x\right] \\
& \leq c(\alpha)\|\nabla \eta\|_{\infty}^{2}\left[\delta \int_{\operatorname{spt} \eta} \Gamma_{\delta}^{\frac{q}{2}} \tilde{\Gamma}_{\delta}^{\frac{\beta}{2}} \mathrm{~d} x+\int_{\operatorname{spt} \eta} \tilde{\Gamma}_{\delta}^{\frac{p+\beta}{2}} \mathrm{~d} x+\int_{\mathrm{spt} \eta}^{\tilde{\Gamma}_{\delta}^{\frac{\beta+2}{2}}} \Gamma_{n, \delta}^{\frac{q-2}{2}} \mathrm{~d} x\right] \\
& +c(\alpha)\left[\delta \int_{B_{R}\left(x_{0}\right)} \eta^{2} \Gamma_{\delta}^{\frac{q}{2}} \tilde{\Gamma}_{\delta}^{\frac{2 \alpha-\beta}{2}} \mathrm{~d} x\right. \\
& \left.\quad+\int_{B_{R}\left(x_{0}\right)} \eta^{2} \tilde{\Gamma}_{\delta}^{\frac{p+2 \alpha-\beta}{2}} \mathrm{~d} x+\int_{B_{R}\left(x_{0}\right)} \eta^{2} \Gamma_{n, \delta}^{\frac{q}{2}} \tilde{\Gamma}_{\delta}^{\frac{2 \alpha-\beta}{2}} \mathrm{~d} x\right],
\end{aligned}
$$

where the last inequality follows from $\left(2.2_{\beta}\right)$. 
At the next step we combine (3.1), (3.2), and (3.4); this yields

$$
\begin{gathered}
\delta \int_{B_{R}\left(x_{0}\right)} \eta^{2} \Gamma_{\delta}^{\frac{q}{2}} \tilde{\Gamma}_{\delta}^{\frac{1+\alpha}{2}} \mathrm{~d} x+\int_{B_{R}\left(x_{0}\right)} \tilde{\Gamma}_{\delta}^{\frac{p+1+\alpha}{2}} \mathrm{~d} x+\int_{B_{R}\left(x_{0}\right)} \tilde{\Gamma}_{\delta}^{\frac{1+\alpha}{2}} \Gamma_{n, \delta}^{\frac{q}{2}} \eta^{2} \mathrm{~d} x \\
\leq c(\alpha)\left[\tau \delta\|\nabla \eta\|_{\infty} \int_{\operatorname{spt} \eta} \Gamma_{\delta}^{\frac{q-2}{2}}\left|\nabla u_{\delta}\right|^{2} \tilde{\Gamma}_{\delta}^{\frac{1+\alpha}{2}} \mathrm{~d} x+c(\tau) \delta\|\nabla \eta\|_{\infty} \int_{\operatorname{spt} \eta} \Gamma_{\delta}^{\frac{q-2}{2}} \tilde{\Gamma}_{\delta}^{\frac{1+\alpha}{2}} \mathrm{~d} x\right. \\
+\tau\|\nabla \eta\|_{\infty} \int_{\operatorname{spt} \eta} \tilde{\Gamma}_{\delta}^{\frac{p+\alpha+1}{2}} \mathrm{~d} x+c(\tau)\|\nabla \eta\|_{\infty} \int_{\operatorname{spt} \eta} \tilde{\Gamma}_{\delta}^{\frac{p+\alpha-1}{2}} \mathrm{~d} x \\
+\tau\|\nabla \eta\|_{\infty} \int_{\operatorname{spt} \eta} \tilde{\Gamma}_{\delta}^{\frac{1+\alpha}{2}} \Gamma_{n, \delta}^{\frac{q}{2}} \mathrm{~d} x+c(\tau)\|\nabla \eta\|_{\infty} \int_{\operatorname{spt} \eta} \tilde{\Gamma}_{\delta}^{\frac{1+\alpha}{2}} \mathrm{~d} x \\
+\|\nabla \eta\|_{\infty}^{2} \delta \int_{\operatorname{spt} \eta} \Gamma_{\delta}^{\frac{q}{2}} \tilde{\Gamma}_{\delta}^{\frac{\beta}{2}} \mathrm{~d} x+\|\nabla \eta\|_{\infty}^{2} \int_{\operatorname{spt} \eta} \tilde{\Gamma}_{\delta}^{\frac{p+\beta}{2}} \mathrm{~d} x \\
+\|\nabla \eta\|_{\infty}^{2} \int_{\operatorname{spt} \eta} \tilde{\Gamma}_{\delta}^{\frac{\beta+2}{2}} \Gamma_{n, \delta}^{\frac{q-2}{2}} \mathrm{~d} x+\delta \int_{\operatorname{spt} \eta} \eta^{2} \Gamma_{\delta}^{\frac{q}{2}} \tilde{\Gamma}_{\delta}^{\frac{2 \alpha-\beta}{2}} \mathrm{~d} x \\
\left.+\int_{\operatorname{spt} \eta}^{\frac{p+2 \alpha-\beta}{2}} \mathrm{~d} x+\int_{\operatorname{spt} \eta} \Gamma_{n, \delta}^{\frac{q}{2}} \tilde{\Gamma}_{\delta}^{\frac{2 \alpha-\beta}{2}} \mathrm{~d} x\right] \\
=: c(\alpha) \sum_{l=1}^{12} T_{l} .
\end{gathered}
$$

From the proof of Theorem 1.1 we already know that the quantities

$$
\tilde{\Gamma}_{\delta}^{\frac{p+1}{2}}, \quad \tilde{\Gamma}_{\delta}^{\frac{1}{2}} \Gamma_{n, \delta}^{\frac{q}{2}}
$$

are uniformly bounded in the space $L_{\text {loc }}^{1}\left(B_{R}\left(x_{0}\right)\right)$; now $(2.15)$ and the uniform boundedness of the right-hand side of (2.16) immediately give the same result for $\delta \Gamma_{\delta}^{q / 2} \tilde{\Gamma}_{\delta}^{1 / 2}$. We define

$$
\alpha_{0}=\beta_{0}=0, \quad \alpha_{i}=\frac{1}{2}+\alpha_{i-1}, \quad \beta_{i}=\alpha_{i-1}, \quad i \in \mathbb{N},
$$

i.e., $\alpha_{i}=i / 2, \beta_{i}=(i-1) / 2$. Then we suppose that, for a suitable constant $c(\rho)$ (also depending on $i$ ), we have

$$
\int_{B_{\rho}\left(x_{0}\right)} \delta \Gamma_{\delta}^{\frac{q}{2}} \tilde{\Gamma}_{\delta}^{\frac{1+\alpha_{i-1}}{2}} \mathrm{~d} x+\int_{B_{\rho}\left(x_{0}\right)}\left[\tilde{\Gamma}_{\delta}^{\frac{p+1+\alpha_{i-1}}{2}}+\tilde{\Gamma}_{\delta}^{\frac{1+\alpha_{i-1}}{2}} \Gamma_{n, \delta}^{\frac{q}{2}}\right] \mathrm{d} x \leq c(\rho)
$$

for any $\rho<R$, and we are going to prove (3.6) with $\alpha_{i-1}$ replaced by $\alpha_{i}$. (Note that by the remarks after (3.5) we already know the validity of $(3.6)$ for $i=1$.)

For this, we choose $s<t<R$ and let $\eta \in C_{0}^{\infty}\left(B_{t}\left(x_{0}\right)\right)$ satisfy $\eta \equiv 1$ on $B_{s}\left(x_{0}\right)$, $|\nabla \eta| \leq c /(t-s)$. Then we apply (3.5) with $\alpha=\alpha_{i}, \beta=\beta_{i}$. The terms $T_{1}, T_{3}, T_{5}$ can be handled easily (see $\S 2)$ if we require that

$$
\tau\|\nabla \eta\|_{\infty} c\left(\alpha_{i}\right)=\frac{1}{2}
$$

Note that with this choice the constants $c(\tau)$ occurring in (3.5) are bounded from above by $c(t-s)^{-\kappa}$ with some suitable exponent $-\kappa$. For $T_{2}$ we observe that, clearly,

$$
\Gamma_{\delta}^{\frac{q-2}{2}} \tilde{\Gamma}_{\delta}^{\frac{1+\alpha_{i}}{2}} \leq \Gamma_{\delta}^{\frac{q}{2}} \tilde{\Gamma}_{\delta}^{\frac{1+\alpha_{i-1}}{2}}
$$

so that $T_{2}$ is bounded by a local constant, due to (3.6). The same is true for $T_{4}$ and $T_{6}$.

Since $\beta_{i}=\alpha_{i-1}<\alpha_{i-1}+1$, there is no problem with $T_{7}$, and since $p+\beta_{i}<$ $p+1+\alpha_{i-1}$, the quantity $T_{8}$ behaves nicely; i.e., we merely replace $\delta \int_{B_{t}\left(x_{0}\right)} \Gamma_{\delta}^{q / 2} \tilde{\Gamma}_{\delta}^{\beta_{i} / 2} \mathrm{~d} x$ 
and $\int_{B_{t}\left(x_{0}\right)} \tilde{\Gamma}_{\delta}^{\left(p+\beta_{i}\right) / 2} \mathrm{~d} x$ by $\delta \int_{B_{t}\left(x_{0}\right)} \Gamma_{\delta}^{q / 2} \tilde{\Gamma}_{\delta}^{\left(1+\alpha_{i-1}\right) / 2} \mathrm{~d} x$ and $\int_{B_{t}\left(x_{0}\right)} \tilde{\Gamma}_{\delta}^{\left(p+1+\alpha_{i-1}\right) / 2} \mathrm{~d} x$, respectively. In order to control $T_{9}$, we estimate

$$
\begin{gathered}
\tilde{\Gamma}_{\delta}^{\frac{\beta_{i}+2}{2}} \Gamma_{n, \delta}^{\frac{q-2}{2}}=\Gamma_{n, \delta}^{\frac{q-2}{2}} \tilde{\Gamma}_{\delta}^{\frac{q-2}{q} \frac{1+\alpha_{i-1}}{2}} \tilde{\Gamma}_{\delta}^{\frac{\beta_{i}+2}{2}-\frac{q-2}{q} \frac{1+\alpha_{i-1}}{2}} \\
\leq \Gamma_{n, \delta}^{\frac{q}{2}} \tilde{\Gamma}_{\delta}^{\frac{1+\alpha_{i-1}}{2}}+\tilde{\Gamma}_{\delta}^{\frac{q}{2}\left[\frac{\beta_{i}+2}{2}-\frac{q-2}{q} \frac{1+\alpha_{i-1}}{2}\right]} .
\end{gathered}
$$

Obviously,

$$
\begin{aligned}
\frac{q}{2}\left[\frac{\beta_{i}+2}{2}-\frac{q-2}{q} \frac{1+\alpha_{i-1}}{2}\right] & \leq \frac{p+1+\alpha_{i-1}}{2} \\
\Longleftrightarrow \frac{q}{2}\left[\frac{q+2+2 \alpha_{i-1}}{2 q}\right] & \leq \frac{p+1+\alpha_{i-1}}{2}
\end{aligned}
$$

(the latter inequality is implied by our requirement $q \leq 2 p$ ). A calculation, using (3.6), further shows that $T_{9}$ is bounded.

Next, we have $2 \alpha_{i}-\beta_{i}=1+\alpha_{i-1}, p+2 \alpha_{i}-\beta_{i}=p+1+\alpha_{i-1}$, whence $T_{10}, T_{11}$ are bounded, again by (3.6).

Referring to (3.6) for the last time, we also get a bound for

$$
\int_{B_{t}\left(x_{0}\right)} \Gamma_{n, \delta}^{q / 2} \tilde{\Gamma}_{\delta}^{\left(2 \alpha_{i}-\beta_{i}\right) / 2} \mathrm{~d} x
$$

Collecting these estimates and returning to (3.5) we see that the inequality

$$
\int_{B_{s}\left(x_{0}\right)}\left[\delta \Gamma_{\delta}^{\frac{q}{2}} \tilde{\Gamma}_{\delta}^{\frac{1+\alpha_{i}}{2}}+\tilde{\Gamma}_{\delta}^{\frac{p+1+\alpha_{i}}{2}}+\Gamma_{n, \delta}^{\frac{q}{2}} \tilde{\Gamma}_{\delta}^{\frac{1+\alpha_{i}}{2}}\right] \mathrm{d} x \leq \frac{1}{2} \int_{B_{t}\left(x_{0}\right)}[\ldots] \mathrm{d} x+A(t-s)^{-\gamma}+B
$$

is valid for $0<s<t \leq \rho<R$, where $A$ and $B$ are local constants depending on $\rho$ and the bounds for the quantity [...] with $\alpha_{i}$ replaced by $\alpha_{i-1}$, but independent of $\delta=\delta(\epsilon)$. As in $\S 2$, the above inequality immediately implies the desired version of (3.6). Since $\alpha_{i} \rightarrow \infty$ as $i \rightarrow \infty$, we have shown that

$$
\int_{B_{\rho}\left(x_{0}\right)} \tilde{\Gamma}_{\delta}^{\frac{t}{2}} \mathrm{~d} x+\int_{B_{\rho}\left(x_{0}\right)} \tilde{\Gamma}_{\delta}^{\frac{t}{2}} \Gamma_{n, \delta}^{\frac{q}{2}} \mathrm{~d} x \leq c(t, \rho)
$$

for any $t>1$ and all radii $\rho<R$, where the constant is independent of $\delta(\epsilon)$. Since $u_{\delta} \rightarrow u$ in $W_{p}^{1}\left(B_{R}\left(x_{0}\right)\right)$ as $\epsilon \rightarrow 0$, it is immediate that $\tilde{\nabla} u \in L_{\text {loc }}^{s}\left(\Omega ; \mathbb{R}^{n-1}\right)$ for any $s<\infty$, whence $\nabla u \in L_{\text {loc }}^{q}\left(\Omega ; \mathbb{R}^{n}\right)$.

\section{§4. IMPROVEMENT OF THE INITIAL HIGHER INTEGRABILITY \\ IN THE SCALAR CASE, AND PROOF OF COROLLARY 1.2}

Using (3.7) and the method of iteration introduced in the previous section, we shall show that

$$
\int_{B_{\rho}\left(x_{0}\right)}\left|\partial_{n} u_{\delta}\right|^{t} \mathrm{~d} x \leq c(t, \rho)<\infty
$$

for any $t<\infty$ and all $\rho<R$. Suppose $\beta \geq 0$ and $\eta \in C_{0}^{\infty}\left(B_{R}\left(x_{0}\right)\right)$. We have

$$
0=\int_{B_{R}\left(x_{0}\right)} D^{2} f_{\delta}\left(\nabla u_{\delta}\right)\left(\partial_{n} \nabla u_{\delta}, \nabla\left[\eta^{2} \partial_{n} u_{\delta} \Gamma_{n, \delta}^{\frac{\beta}{2}}\right]\right) \mathrm{d} x
$$

and, since we deal with the scalar case, this implies

$$
\begin{aligned}
& \int_{B_{R}\left(x_{0}\right)} D^{2} f_{\delta}\left(\nabla u_{\delta}\right)\left(\partial_{n} \nabla u_{\delta}, \partial_{n} \nabla u_{\delta}\right) \eta^{2} \Gamma_{n, \delta}^{\frac{\beta}{2}} \mathrm{~d} x \\
& \quad \leq c \int_{B_{R}\left(x_{0}\right)} D^{2} f_{\delta}\left(\nabla u_{\delta}\right)(\nabla \eta, \nabla \eta)\left|\partial_{n} u_{\delta}\right|^{2} \Gamma_{n, \delta}^{\frac{\beta}{2}} \mathrm{~d} x .
\end{aligned}
$$


As before, here we have used the Cauchy-Schwarz and the Young inequality. The structure of $D^{2} f_{\delta}$ gives the estimate

$$
\begin{aligned}
& \delta \int_{B_{R}\left(x_{0}\right)} \eta^{2} \Gamma_{\delta}^{\frac{q-2}{2}} \Gamma_{n, \delta}^{\frac{\beta}{2}}\left|\partial_{n} \nabla u_{\delta}\right|^{2} \mathrm{~d} x+\int_{B_{R}\left(x_{0}\right)} \eta^{2} \tilde{\Gamma}_{\delta}^{\frac{p-2}{2}} \Gamma_{n, \delta}^{\frac{\beta}{2}}\left|\partial_{n} \tilde{\nabla} u_{\delta}\right|^{2} \mathrm{~d} x \\
& +\int_{B_{R}\left(x_{0}\right)} \eta^{2} \Gamma_{n, \delta}^{\frac{q-2}{2}+\frac{\beta}{2}}\left|\partial_{n} \partial_{n} u_{\delta}\right|^{2} \mathrm{~d} x \\
& \quad \leq c\|\nabla \eta\|_{\infty}^{2}\left[\int_{\operatorname{spt} \eta} \delta \Gamma_{\delta}^{\frac{q-2}{2}} \Gamma_{n, \delta}^{\frac{\beta}{2}+1} \mathrm{~d} x+\int_{\operatorname{spt} \eta} \tilde{\Gamma}_{\delta}^{\frac{p-2}{2}} \Gamma_{n, \delta}^{\frac{\beta}{2}+1} \mathrm{~d} x+\int_{\operatorname{spt} \eta} \Gamma_{n, \delta}^{\frac{q+\beta}{2}} \mathrm{~d} x\right] .
\end{aligned}
$$

Next, we return to (2.4), choosing $\varphi=\eta^{2} u_{\delta} \Gamma_{n, \delta}^{(1+\alpha) / 2}, \alpha \geq 0$. We get

$$
\begin{aligned}
& \int_{B_{R}\left(x_{0}\right)} \eta^{2} \Gamma_{n, \delta}^{\frac{1+\alpha}{2}} D f_{\delta}\left(\nabla u_{\delta}\right) \cdot \nabla u_{\delta} \mathrm{d} x \\
& =-2 \int_{B_{R}\left(x_{0}\right)} \eta \nabla \eta \cdot D f_{\delta}\left(\nabla u_{\delta}\right) u_{\delta} \Gamma_{n, \delta}^{\frac{1+\alpha}{2}} \mathrm{~d} x \\
& \quad-(1+\alpha) \int_{B_{R}\left(x_{0}\right)} u_{\delta} \eta^{2} D f_{\delta}\left(\nabla u_{\delta}\right) \cdot \nabla\left(\partial_{n} u_{\delta}\right) \Gamma_{n, \delta}^{\frac{\alpha-1}{2}} \partial_{n} u_{\delta} \mathrm{d} x
\end{aligned}
$$

By (2.6),

the left-hand side of (4.3)

$$
\begin{aligned}
\geq c\left[\int_{B_{R}\left(x_{0}\right)} \delta \eta^{2} \Gamma_{\delta}^{\frac{q-2}{2}}\left|\nabla u_{\delta}\right|^{2} \Gamma_{n, \delta}^{\frac{1+\alpha}{2}} \mathrm{~d} x+\right. & \int_{B_{R}\left(x_{0}\right)} \eta^{2} \tilde{\Gamma}_{\delta}^{\frac{p-2}{2}}\left|\tilde{\nabla} u_{\delta}\right|^{2} \Gamma_{n, \delta}^{\frac{1+\alpha}{2}} \mathrm{~d} x \\
& \left.+\int_{B_{R}\left(x_{0}\right)} \eta^{2} \Gamma_{n, \delta}^{\frac{q-2}{2}+\frac{1+\alpha}{2}}\left|\partial_{n} u_{\delta}\right|^{2} \mathrm{~d} x\right],
\end{aligned}
$$

and moreover, (2.7) shows that

|the first term on the right in (4.3)|

$$
\begin{aligned}
\leq c\left[\int_{B_{R}\left(x_{0}\right)} \eta|\nabla \eta| \delta \Gamma_{\delta}^{\frac{q-2}{2}}\left|\nabla u_{\delta}\right| \Gamma_{n, \delta}^{\frac{1+\alpha}{2}} \mathrm{~d} x\right. & +\int_{B_{R}\left(x_{0}\right)} \eta|\nabla \eta| \tilde{\Gamma}_{\delta}^{\frac{p-2}{2}}\left|\tilde{\nabla} u_{\delta}\right| \Gamma_{n, \delta}^{\frac{1+\alpha}{2}} \mathrm{~d} x \\
& \left.+\int_{B_{R}\left(x_{0}\right)} \eta|\nabla \eta| \Gamma_{n, \delta}^{\frac{q-2}{2}+\frac{1+\alpha}{2}}\left|\partial_{n} u_{\delta}\right| \mathrm{d} x\right] \\
\leq c\left[\tau \int_{B_{R}\left(x_{0}\right)} \delta \eta^{2} \Gamma_{\delta}^{\frac{q-2}{2}}\left|\nabla u_{\delta}\right|^{2} \Gamma_{n, \delta}^{\frac{1+\alpha}{2}} \mathrm{~d} x\right. & +\frac{1}{\tau} \int_{B_{R}\left(x_{0}\right)} \delta|\nabla \eta|^{2} \Gamma_{\delta}^{\frac{q-2}{2}} \Gamma_{n, \delta}^{\frac{1+\alpha}{2}} \mathrm{~d} x \\
& +\tau \int_{B_{R}\left(x_{0}\right)} \eta^{2} \tilde{\Gamma}_{\delta}^{\frac{p-2}{2}}\left|\tilde{\nabla} u_{\delta}\right|^{2} \Gamma_{n, \delta}^{\frac{1+\alpha}{2}} \mathrm{~d} x+\frac{1}{\tau} \int_{B_{R}\left(x_{0}\right)}|\nabla \eta|^{2} \tilde{\Gamma}_{\delta}^{\frac{p-2}{2}} \Gamma_{n, \delta}^{\frac{1+\alpha}{2}} \mathrm{~d} x \\
& \left.+\tau \int_{B_{R}\left(x_{0}\right)} \eta^{2} \Gamma_{n, \delta}^{\frac{q-2}{2}}\left|\partial_{n} u_{\delta}\right|^{2} \Gamma_{n, \delta}^{\frac{1+\alpha}{2}} \mathrm{~d} x+\frac{1}{\tau} \int_{B_{R}\left(x_{0}\right)}|\nabla \eta|^{2} \Gamma_{n, \delta}^{\frac{q-2}{2}+\frac{1+\alpha}{2}} \mathrm{~d} x\right]
\end{aligned}
$$

for $\tau \in(0,1)$. If we use this estimate and choose $\tau$ sufficiently small, then the $\tau$-terms can be absorbed in the left-hand side; more precisely, they can be absorbed in the terms 
giving the lower bound stated in (4.4). This yields

$$
\begin{aligned}
& \int_{B_{R}\left(x_{0}\right)} \delta \eta^{2} \Gamma_{\delta}^{\frac{q-2}{2}}\left|\nabla u_{\delta}\right|^{2} \Gamma_{n, \delta}^{\frac{1+\alpha}{2}} \mathrm{~d} x+\int_{B_{R}\left(x_{0}\right)} \eta^{2} \tilde{\Gamma}_{\delta}^{\frac{p-2}{2}}\left|\tilde{\nabla} u_{\delta}\right|^{2} \Gamma_{n, \delta}^{\frac{1+\alpha}{2}} \mathrm{~d} x \\
& +\int_{B_{R}\left(x_{0}\right)} \eta^{2} \Gamma_{n, \delta}^{\frac{q-1+\alpha}{2}}\left|\partial_{n} u_{\delta}\right|^{2} \mathrm{~d} x \\
& \quad \leq c\|\nabla \eta\|_{\infty}^{2}\left[\int_{\text {spt } \eta} \delta \Gamma_{\delta}^{\frac{q-2}{2}} \Gamma_{n, \delta}^{\frac{1+\alpha}{2}} \mathrm{~d} x+\int_{\text {spt } \eta} \tilde{\Gamma}_{\delta}^{\frac{p-2}{2}} \Gamma_{n, \delta}^{\frac{1+\alpha}{2}} \mathrm{~d} x+\int_{\operatorname{spt} \eta} \Gamma_{n, \delta}^{\frac{q-1+\alpha}{2}} \mathrm{~d} x\right] \\
& \quad+c \mid \text { the second term on the right in }(4.3) \mid .
\end{aligned}
$$

To estimate the second term on the right in (4.3), we observe that (cf. (2.11))

$$
\begin{aligned}
& \left|D f_{\delta}\left(\nabla u_{\delta}\right) \cdot \nabla\left(\partial_{n} u_{\delta}\right)\right| \\
& \quad \leq c\left[\delta \Gamma_{\delta}^{\frac{q-2}{2}}\left|\nabla u_{\delta}\right|\left|\partial_{n} \nabla u_{\delta}\right|+\tilde{\Gamma}_{\delta}^{\frac{p-2}{2}}\left|\tilde{\nabla} u_{\delta}\right|\left|\partial_{n} \tilde{\nabla} u_{\delta}\right|+\Gamma_{n, \delta}^{\frac{q-2}{2}}\left|\partial_{n} u_{\delta}\right|\left|\partial_{n} \partial_{n} u_{\delta}\right|\right] .
\end{aligned}
$$

Thus, by (4.2),

|the second term on the right in (4.3)|

$$
\begin{aligned}
& \leq c(\alpha)\left[\int_{B_{R}\left(x_{0}\right)} \delta \eta^{2} \Gamma_{\delta}^{\frac{q-1}{2}}\left|\partial_{n} \nabla u_{\delta}\right| \Gamma_{n, \delta}^{\frac{\alpha}{2}} \mathrm{~d} x\right. \\
& \left.\quad+\int_{B_{R}\left(x_{0}\right)} \eta^{2} \tilde{\Gamma}_{\delta}^{\frac{p-1}{2}}\left|\partial_{n} \tilde{\nabla} u_{\delta}\right| \Gamma_{n, \delta}^{\frac{\alpha}{2}} \mathrm{~d} x+\int_{B_{R}\left(x_{0}\right)} \eta^{2} \Gamma_{n, \delta}^{\frac{q-1}{2}}\left|\partial_{n} \partial_{n} u_{\delta}\right| \Gamma_{n, \delta}^{\frac{\alpha}{2}} \mathrm{~d} x\right] \\
& \leq c(\alpha)\left[\int_{B_{R}\left(x_{0}\right)} \delta \eta^{2} \Gamma_{\delta}^{\frac{q-2}{2}} \Gamma_{n, \delta}^{\frac{\beta}{2}}\left|\partial_{n} \nabla u_{\delta}\right|^{2} \mathrm{~d} x+\int_{B_{R}\left(x_{0}\right)} \eta^{2} \tilde{\Gamma}_{\delta}^{\frac{p-2}{2}} \Gamma_{\delta}^{\frac{\beta}{2}}\left|\partial_{n} \tilde{\nabla} u_{\delta}\right|^{2} \mathrm{~d} x\right. \\
& \quad+\int_{B_{R}\left(x_{0}\right)} \eta^{2} \Gamma_{n, \delta}^{\frac{q-2}{2}+\frac{\beta}{2}}\left|\partial_{n} \partial_{n} u_{\delta}\right|^{2} \mathrm{~d} x+\int_{B_{R}\left(x_{0}\right)} \delta \eta^{2} \Gamma_{\delta}^{\frac{q}{2}} \Gamma_{n, \delta}^{\frac{2 \alpha-\beta}{2}} \mathrm{~d} x \\
& \left.\quad+\int_{B_{R}\left(x_{0}\right)} \eta^{2} \tilde{\Gamma}_{\delta}^{\frac{p}{2}} \Gamma_{n, \delta}^{\frac{2 \alpha-\beta}{2}} \mathrm{~d} x+\int_{B_{R}\left(x_{0}\right)} \eta^{2} \Gamma_{n, \delta}^{\frac{q}{2}} \Gamma_{n, \delta}^{\frac{2 \alpha-\beta}{2}} \mathrm{~d} x\right] \\
& \leq c(\alpha)\|\nabla \eta\|_{\infty}^{2}\left[\int_{\operatorname{spt} \eta} \delta \Gamma_{\delta}^{\frac{q-2}{2}} \Gamma_{n, \delta}^{\frac{\beta}{2}+1} \mathrm{~d} x+\int_{\operatorname{spt} \eta} \tilde{\Gamma}_{\delta}^{\frac{p-2}{2}} \Gamma_{n, \delta}^{\frac{\beta}{2}+1} \mathrm{~d} x+\int_{\operatorname{spt} \eta}^{\Gamma_{n, \delta}^{\frac{q+\beta}{2}}} \mathrm{~d} x\right] \\
& \quad c(\alpha)\left[\int_{\operatorname{spt} \eta} \delta \Gamma_{\delta}^{\frac{q}{2}} \Gamma_{n, \delta}^{\frac{2 \alpha-\beta}{2}} \mathrm{~d} x+\int_{\operatorname{spt} \eta} \tilde{\Gamma}_{\delta}^{\frac{p}{2}} \Gamma_{n, \delta}^{\frac{2 \alpha-\beta}{2}} \mathrm{~d} x+\int_{\operatorname{spt} \eta} \Gamma_{n, \delta}^{\frac{q+2 \alpha-\beta}{2}} \mathrm{~d} x\right] .
\end{aligned}
$$

We plug this estimate in (4.5) (observing at the same time that the quantities such as $\Gamma_{\delta}^{(q-2) / 2}\left|\nabla u_{\delta}\right|^{2}$ occurring on the left in (4.5) can be replaced by $\Gamma_{\delta}^{q / 2}$, because the resulting difference is already involved in the expression on the right-hand side of (4.5)), obtaining

$$
\begin{aligned}
& \int_{B_{R}\left(x_{0}\right)} \delta \eta^{2} \Gamma_{\delta}^{\frac{q}{2}} \Gamma_{n, \delta}^{\frac{1+\alpha}{2}} \mathrm{~d} x+\int_{B_{R}\left(x_{0}\right)} \eta^{2} \tilde{\Gamma}_{\delta}^{\frac{p}{2}} \Gamma_{n, \delta}^{\frac{1+\alpha}{2}} \mathrm{~d} x+\int_{B_{R}\left(x_{0}\right)} \eta^{2} \Gamma_{n, \delta}^{\frac{q+1+\alpha}{2}} \mathrm{~d} x \\
& \leq c(\alpha)\|\nabla \eta\|_{\infty}^{2}\left[\int_{\operatorname{spt} \eta} \delta \Gamma_{\delta}^{\frac{q-2}{2}}\left[\Gamma_{n, \delta}^{\frac{1+\alpha}{2}}+\Gamma_{n, \delta}^{\frac{2+\beta}{2}}\right] \mathrm{d} x\right. \\
& \left.+\int_{\operatorname{spt} \eta} \tilde{\Gamma}_{\delta}^{\frac{p-2}{2}}\left[\Gamma_{n, \delta}^{\frac{1+\alpha}{2}}+\Gamma_{n, \delta}^{\frac{2+\beta}{2}}\right] \mathrm{d} x+\int_{\operatorname{spt} \eta}\left[\Gamma_{n, \delta}^{\frac{q-1+\alpha}{2}}+\Gamma_{n, \delta}^{\frac{q+\beta}{2}}\right] \mathrm{d} x\right] \\
& +c(\alpha)\left[\int_{\operatorname{spt} \eta} \delta \Gamma_{\delta}^{\frac{q}{2}} \Gamma_{n, \delta}^{\frac{2 \alpha-\beta}{2}} \mathrm{~d} x+\int_{\operatorname{spt} \eta} \tilde{\Gamma}_{\delta}^{\frac{p}{2}} \Gamma_{n, \delta}^{\frac{2 \alpha-\beta}{2}} \mathrm{~d} x+\int_{\operatorname{spt} \eta} \Gamma_{n, \delta}^{\frac{q+2 \alpha-\beta}{2}} \mathrm{~d} x\right] .
\end{aligned}
$$


Now we use this inequality with $\alpha_{0}=0, \beta_{0}=0, \alpha_{i}=\alpha_{i-1}+1 / 2, \beta_{i}=\alpha_{i-1}, i \geq 1$; in particular, we have $1+\beta_{i} / 2=\frac{1}{2}\left(\alpha_{i}+\frac{3}{2}\right)$. As a result, for $i \geq 1$,

$$
\begin{aligned}
& \int_{B_{R}\left(x_{0}\right)} \delta \eta^{2} \Gamma_{\delta}^{\frac{q}{2}} \Gamma_{n, \delta}^{\frac{1+\alpha_{i}}{2}} \mathrm{~d} x+\int_{B_{R}\left(x_{0}\right)} \eta^{2} \tilde{\Gamma}_{\delta}^{\frac{p}{2}} \Gamma_{n, \delta}^{\frac{1+\alpha_{i}}{2}} \mathrm{~d} x+\int_{B_{R}\left(x_{0}\right)} \eta^{2} \Gamma_{n, \delta}^{\frac{q+1+\alpha_{i}}{2}} \mathrm{~d} x \\
& \leq c(i)\|\nabla \eta\|_{\infty}^{2}\left[\int_{\operatorname{spt} \eta} \delta \Gamma_{\delta}^{\frac{q-2}{2}} \Gamma_{n, \delta}^{\frac{\alpha_{i}+3 / 2}{2}} \mathrm{~d} x+\int_{\operatorname{spt} \eta} \tilde{\Gamma}_{\delta}^{\frac{p-2}{2}} \Gamma_{n, \delta}^{\frac{\alpha_{i}+3 / 2}{2}} \mathrm{~d} x+\int_{\operatorname{spt} \eta} \Gamma_{n, \delta}^{\frac{q+\beta_{i}}{2}} \mathrm{~d} x\right] \\
& \quad+c(i)\left[\int_{\operatorname{spt} \eta} \delta \Gamma_{\delta}^{\frac{q}{2}} \Gamma_{n, \delta}^{\frac{2 \alpha_{i}-\beta_{i}}{2}} \mathrm{~d} x+\int_{\operatorname{spt} \eta} \tilde{\Gamma}_{\delta}^{\frac{p}{2}} \Gamma_{n, \delta}^{\frac{2 \alpha_{i}-\beta_{i}}{2}} \mathrm{~d} x+\int_{\operatorname{spt} \eta} \Gamma_{n, \delta}^{\frac{q+2 \alpha_{i}-\beta_{i}}{2}} \mathrm{~d} x\right] .
\end{aligned}
$$

We claim that for all $i \in \mathbb{N}_{0}$ and for any radius $\rho<R$ we have

$$
\left(4.7_{i}\right) \quad \int_{B_{\rho}\left(x_{0}\right)} \delta \Gamma_{\delta}^{\frac{q}{2}} \Gamma_{n, \delta}^{\frac{1+\alpha_{i}}{2}} \mathrm{~d} x+\int_{B_{\rho}\left(x_{0}\right)} \tilde{\Gamma}_{\delta}^{\frac{p}{2}} \Gamma_{n, \delta}^{\frac{1+\alpha_{i}}{2}} \mathrm{~d} x+\int_{B_{\rho}\left(x_{0}\right)} \Gamma_{n, \delta}^{\frac{q+1+\alpha_{i}}{2}} \mathrm{~d} x \leq c(i, \rho)<\infty .
$$

Let $i=0$. Then, obviously, the last four integrals on the right in $\left(4.6_{0}\right)$ are bounded independently of $\delta$, for the second integral we use (3.7), and for the first we estimate

$$
\begin{aligned}
\Gamma_{\delta}^{\frac{q-2}{2}} \Gamma_{\delta, n}^{\frac{3}{4}} & \leq c\left[\tilde{\Gamma}_{\delta}^{\frac{q-2}{2}} \Gamma_{\delta, n}^{\frac{3}{4}}+\Gamma_{n, \delta}^{\frac{q-2}{2}} \Gamma_{n, \delta}^{\frac{3}{4}}\right] \\
& \leq c\left[\tilde{\Gamma}_{\delta}^{\frac{q-2}{2}} \Gamma_{\delta, n}^{\frac{q}{2}}+\Gamma_{n, \delta}^{\frac{q}{2}}\right]
\end{aligned}
$$

so that (3.7) applies again. Thus the left-hand side of $\left(4.6_{0}\right)$ remains bounded independently of $\delta$, and we get (4.70). Now, suppose that $\left(4.7_{i-1}\right)$ is true for some $i \geq 1$. Then the integrals on the right in $\left(4.6_{i}\right)$ can be bounded independently of $\delta$, because the following estimates are true:

$$
\begin{aligned}
& \delta \Gamma_{\delta}^{\frac{q-2}{2}} \Gamma_{n, \delta}^{\frac{\alpha_{i}+3 / 2}{2}} \\
& \quad \leq \delta \Gamma_{\delta}^{\frac{q}{2}} \Gamma_{n, \delta}^{\frac{\alpha_{i}+3 / 2}{2}-1}=\delta \Gamma_{\delta}^{\frac{q}{2}} \Gamma_{n, \delta}^{\frac{\alpha_{i}-1 / 2}{2^{2}}}=\delta \Gamma_{\delta}^{\frac{q}{2}} \Gamma_{n, \delta}^{\frac{\alpha_{i-1}}{2}},
\end{aligned}
$$

so that we can use $\left(4.7_{i-1}\right)$. We have

$$
\tilde{\Gamma}_{\delta}^{\frac{p-2}{2}} \Gamma_{n, \delta}^{\frac{\alpha_{i}+3 / 2}{2}} \leq c_{s}\left[\tilde{\Gamma}_{\delta}^{s \frac{p-2}{2}}+\Gamma_{n, \delta}^{\frac{s}{s-1} \frac{\alpha_{i}+3 / 2}{2}}\right]
$$

for any exponent $s>1$ (by the Young inequality) and if " $s \rightarrow \infty$ ", then

$$
\begin{aligned}
\frac{s}{s-1} & \frac{1}{2}\left(\alpha_{i}+\frac{3}{2}\right)=\frac{s}{s-1} \frac{1}{2}\left(\alpha_{i-1}+2\right) \\
& <\frac{1}{2}\left(\alpha_{i-1}+q+1\right),
\end{aligned}
$$

which allows us to use $\left(4.7_{i}\right)$ for the $\Gamma_{n, \delta}$-part, whereas $\tilde{\Gamma}_{\delta}^{s \frac{p-2}{2}}$ is handled with the help of the inequality (3.7). Next, we have

$$
\Gamma_{n, \delta}^{\frac{q+\beta_{i}}{2}}=\Gamma_{n, \delta}^{\frac{q+\alpha_{i-1}}{2}} \leq \Gamma_{n, \delta}^{\frac{q+\alpha_{i-1}+1}{2}} .
$$

Thus, again we can refer to $\left(4.7_{i-1}\right)$.

$$
\delta \Gamma_{\delta}^{\frac{q}{2}} \Gamma_{n, \delta}^{\frac{2 \alpha_{i}-\beta_{i}}{2}}=\delta \Gamma_{\delta}^{\frac{q}{2}} \Gamma_{n, \delta}^{\frac{\alpha_{i-1}+1}{2}},
$$

and $\left(4.7_{i-1}\right)$ applies. We observe that

$$
\tilde{\Gamma}^{\frac{p}{2}} \Gamma_{n, \delta}^{\frac{2 \alpha_{i}-\beta_{i}}{\delta^{2}}}=\tilde{\Gamma}^{\frac{p}{2}} \Gamma_{n, \delta}^{\frac{\alpha_{i-1}+1}{2}},
$$

and this term also occurs in $\left(4.7_{i-1}\right)$. The same is true for

$$
\Gamma_{n, \delta}^{\frac{1}{2}\left(q+2 \alpha_{i}-\beta_{i}\right)}=\Gamma_{n, \delta}^{\frac{1}{2}\left(q+\alpha_{i-1}+1\right)} .
$$


Summing (4.8)-(4.13), we see that all the quantities on the right in $\left(4.6_{i}\right)$ are bounded in an appropriate way, which gives $\left(4.7_{i}\right)$; in particular, we have

$$
\int_{B_{\rho}\left(x_{0}\right)} \Gamma_{n, \delta}^{\frac{q+1+\alpha_{i}}{2}} \mathrm{~d} x \leq c(i, \rho)<\infty
$$

for any $i$ and any $\rho<R$. Since $\alpha_{i} \rightarrow \infty$ as $i \rightarrow \infty$, the claim follows, because now we know that

$$
\left\|u_{\delta}\right\|_{W_{t}^{1}\left(B_{\rho}\left(x_{0}\right)\right)} \leq c(\rho, t)
$$

for all $t<\infty, \rho<R$.

With (4.14) at hand, the proof of $C^{1, \alpha}$-regularity can be obtained by following, e.g., [Bi, proof of Theorem 5.22] or [BF4, Lemma 2.9], where it is shown that (4.14) implies $\left\|\nabla u_{\delta}\right\|_{L^{\infty}\left(B_{\rho}\left(x_{0}\right)\right)} \leq c(\rho)<\infty$. Then, uniform Hölder continuity of $\nabla u_{\delta}$ follows from [LU, Chapter $4, \S 6]$.

Acknowledgments. The authors thank N. N. Ural'tseva for many useful discussions.

\section{REFERENCES}

[AF] E. Acerbi and N. Fusco, Partial regularity under anisotropic $(p, q)$ growth conditions, J. Differential Equations 107 (1994), no. 1, 46-67. MR1260848 (95c:49059)

[Bi] M. Bildhauer, Convex variational problems. Linear, nearly linear and anisotropic growth conditions, Lecture Notes in Math., vol. 1818, Springer-Verlag, Berlin, 2003. MR1998189 (2004i:49072)

[BF1] M. Bildhauer and M. Fuchs, Partial regularity for variational integrals with $(s, \mu, q)$-growth, Calc. Var. Partial Differential Equations 13 (2001), 537-560. MR1867941 (2002h:49057)

[BF2] , Two-dimensional anisotropic variational problems, Calc. Var. Partial Differential Equations 16 (2003), 177-186. MR1956853 (2004e:49056)

[BF3] Partial regularity for a class of anisotropic variational integrals with convex hull property, Asymptot. Anal. 32 (2002), 293-315. MR1993652 (2004k:49085)

[BF4],$C^{1, \alpha}$-solutions to non-autonomous anisotropic variational problems, Calc. Var. Partial Differential Equations 24 (2005), 309-340. MR2174429 (2006k:49101)

[BFM] M. Bildhauer, M. Fuchs, and G. Mingione, A priori gradient bounds and local $C^{1, \alpha}$-estimates for (double) obstacle problems under non-standard growth conditions, Z. Anal. Anwendungen 20 (2001), 959-985. MR1884515 (2003d:49052)

[Ca] S. Campanato, Hölder continuity of the solutions of some non-linear elliptic systems, Adv. in Math. 48 (1983), 16-43. MR0697613 (84g:35049)

[Ch] H. J. Choe, Interior behaviour of minimizers for certain functionals with nonstandard growth, Nonlinear Anal. 19 (1992), 933-945. MR1192273 (93k:49002)

[ELM1] L. Esposito, F. Leonetti, and G. Mingione, Regularity results for minimizers of irregular integrals with $(p, q)$-growth, Forum Math. 14 (2002), 245-272. MR1880913 (2003a:49048)

[ELM2] _ Regularity for minimizers of functionals with $p$-q growth, NODEA Nonlinear Differential Equations Appl. 6 (1999), 133-148. MR1694803 (2000h:49056)

[FS] N. Fusco and C. Sbordone, Some remarks on the regularity of minima of anisotropic integrals, Comm. Partial Differential Equations 18 (1993), 153-167. MR1211728 (94e:49013)

[Gi1] M. Giaquinta, Multiple integrals in the calculus of variations and nonlinear elliptic systems, Ann. of Math. Stud., vol. 105, Princeton Univ. Press, Princeton, NJ, 1983. MR0717034 (86b:49003)

[Gi2] - Growth conditions and regularity, a counterexample, Manuscripta Math. 59 (1987), 245-248. MR0905200 (88h:49034)

[GM] M. Giaquinta and G. Modica, Remarks on the regularity of the minimizers of certain degenerate functionals, Manuscripta Math. 57 (1986), 55-99. MR0866406 (87m:49036)

[Ho] M. C. Hong, Some remarks on the minimizers of variational integrals with nonstandard growth conditions, Boll. Un. Mat. Ital. A (7) 6 (1992), 91-101. MR1164739 (93f:49034)

[Ma1] P. Marcellini, Regularity of minimizers of integrals of the calculus of variations with nonstandard growth conditions, Arch. Rational Mech. Anal. 105 (1989), 267-284. MR0969900 (90a:49017)

[Ma2] - Regularity and existence of solutions of elliptic equations with $(p, q)$-growth conditions, J. Differential Equations 90 (1991), 1-30. MR1094446 (92e:35061) 
[Mo] C. B. Morrey, Multiple integrals in the calculus of variations, Grundlehren Math. Wiss., Bd. 130, Springer-Verlag, New York, 1966. MR0202511 (34:2380)

[LU] O. A. Ladyzhenskaya and N. N. Ural'tseva, Linear and quasilinear elliptic equations, "Nauka", Moscow, 1964; English transl., Acad. Press, New York-London, 1968. MR0244627 (39:5941)

[UU] N. N. Ural'tseva and A. B. Urdaletova, The boundedness of the gradients of generalized solutions of degenerate quasilinear nonuniformly elliptic equations, Vestnik Leningrad. Univ. Mat. Mekh. Astronom. 1983, vyp. 4, 50-56; English transl., Vestnik Leningrad Univ. Math. 16 (1984), 263270. MR0725829 (85g:35048)

Department of Mathematics, Saarland University, P.O. Box 1511 50, D-66041 SaArbrücken, Germany

E-mail address: bibi@math.uni-sb.de

Department of Mathematics, SaArland University, P.O. Box 1511 50, D-66041 SaArbrücken, GERMANY

E-mail address: fuchs@math.uni-sb.de

Department of Mathematics and Statistics, University of Jyväskylä, P.O. Box 35, Finland

E-mail address: zhong@maths.jyu.fi

Received 22/APR/2006

Originally published in English 